\title{
Performance of Dual-Polarization QPSK for Optical Transport Systems
}

\author{
Kim Roberts, Member, IEEE, Maurice O'Sullivan, Kuang-Tsan Wu, Member, IEEE, Han Sun, Ahmed Awadalla,
} David J. Krause, Member, IEEE, and Charles Laperle, Member, IEEE

(Invited Paper)

\begin{abstract}
The emergence of capable semiconductor processes has allowed digital signal processing to extend the application range of high-capacity optical systems. We report the performance of polarization multiplexed (or dual-polarization) quadrature phase-shift keying at 40 and $100 \mathrm{~Gb} / \mathrm{s}$.
\end{abstract}

Index Terms-Electronic signal processing, optical coherent transceiver, polarization multiplexing, quadrature phase-shift keying (QPSK).

\section{INTRODUCTION}

O INCE 1980, the growth of fiber network bandwidth demand has been met by achieving increased spectral efficiency through increases in line rate [via time division multiplexing (TDM)] and increases in the number of carriers (via dense wavelength division multiplexing (DWDM)]. Cost reduction has been driven in each generation by increasing the number of bits transported per electro-optic device and per optical amplifier.

However, the physical impairments due to optical filtering, chromatic dispersion, and polarization mode dispersion (PMD) increase so strongly as a function of reach and TDM rate that only a small portion of desired routes are able to support TDM rates of $40 \mathrm{~Gb} / \mathrm{s}$ or above.

Optical transmission has traditionally been based on intensity modulation and direct detection. Direct detection uses a photocurrent that is proportional to the squared magnitude of the complex electric field of the optical signal. However, the optical propagation impairments are predominantly linear functions of that electric field, and the squaring converts a simple linear remediation task in the receiver into a very challenging nonlinear task. At the transmitter, only the intensity (field amplitude) is controlled while phase is at best bounded to contain unruliness.

Within this tradition, equalization of optical dispersion is commonly implemented in the optical domain using dispersion compensating fiber. In demanding applications such as 40 and $10 \mathrm{~Gb} / \mathrm{s}$ ultra long haul a final stage of dispersion compensation tuning is often required, using optical filter compensators. The

Manuscript received January 23, 2009; revised April 27, 2009. First published May 08, 2009; current version published July 24, 2009.

The authors are with Nortel, Ottawa, ON K2H 8E9, Canada (e-mail: krob@nortel.com; osullms@nortel.com; ktwu@nortel.com; hsun1@nortel. com; ahmedaw@nortel.com; davidkr@nortel.com; claperle@nortel.com)

Color versions of one or more of the figures in this paper are available online at http://ieeexplore.ieee.org.

Digital Object Identifier 10.1109/JLT.2009.2022484 loss attendant to such optical domain remedies is compensated with additional optical amplification.

Coherent detection offers the advantage of access to the amplitude and phase of the optical electric field in the electronic domain at the receiver [1], [2]. This allows digital linear filters to compensate the linear channel transfer functions [3]. Chromatic dispersion can be inverted without penalty. The polarization transfer function of the optical link can be tracked allowing electronic domain compensation of PMD and polarization dependent loss (PDL) [4], [5]. This tracking also allows practical polarization multiplexed products [6]. Detection of phase and amplitude enables a wealth of advanced modulation options which trade off spectral efficiency with reach and impairment tolerance. In phase modulated systems, complete compensation of chromatic dispersion at the ends of the link produces increased tolerance to the degradations of cross-phase modulation (XPM) occurring along the optical link.

Here we present performance and design considerations of 40 and $100 \mathrm{~Gb} / \mathrm{s}$ transceivers, implemented with polarization multiplexed, a.k.a. dual polarization, quadrature phase-shift keying (DP-QPSK) modulation and coherent detection architectures, for optical transmission on existing fiber plants. We begin with a comparison of various modulation formats to achieve a line rate of $46 \mathrm{~Gb} / \mathrm{s}$, which includes forward error correction (FEC) overhead. It is shown that with additive white Gaussian noise from amplified spontaneous emission DP-QPSK is the best choice by measure of optical noise tolerance and implementation complexity. We then describe a practical implementation and examine the details of the tolerance to laser phase noise, chromatic dispersion, PMD, PDL, and optical filtering. Finally we explain the tolerance to inter-channel XPM and show that best performance is achieved with complete electronic-domain dispersion compensation. We consider two implementations of $100 \mathrm{~Gb} / \mathrm{s}$, both based on DP-QPSK architecture and both achieving a network spectral efficiency of $2 \mathrm{~b} / \mathrm{s} / \mathrm{Hz}$. The first is a dual sub-carrier implementation where each carrier delivers a $50 \mathrm{~Gb} / \mathrm{s}$ payload. In this scheme coherent detection allows close proximity of the sub-carriers and, therefore, compatibility with a $50-\mathrm{GHz}$ spaced network channelization. In the second scheme, a single carrier is used.

\section{COMPARison OF MOdulation Formats}

We compare the ideal performance of selected modulation formats in the presence of additive white Gaussian noise. The modulation formats of Table I have been scaled to achieve the 


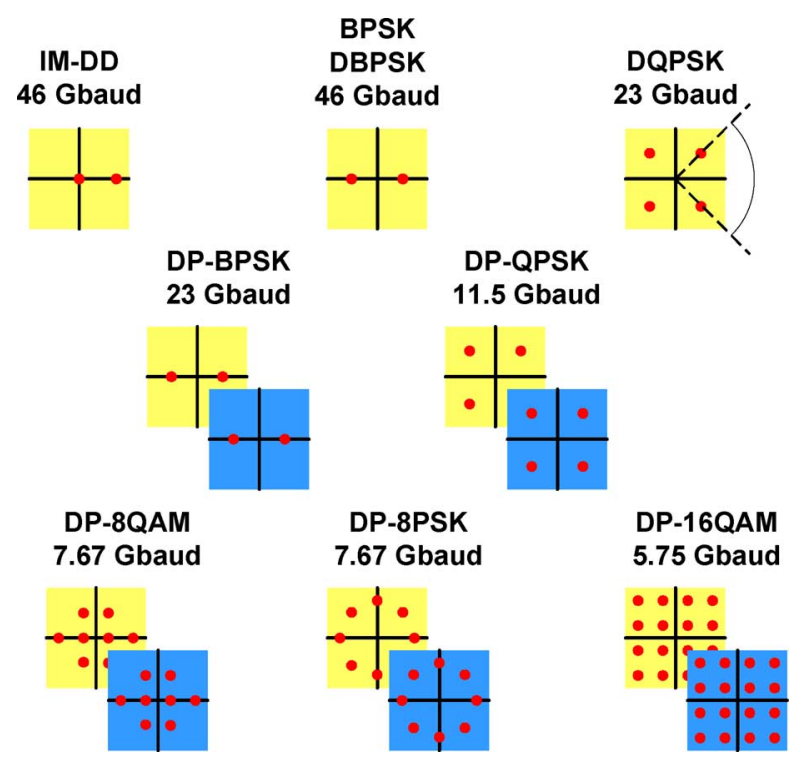

Fig. 1. Constellations of various modulation formats at $46 \mathrm{~Gb} / \mathrm{s}$.

TABLE I

COMPARISON OF MODULATION FORMATS FOR LINE RATE OF $46 \mathrm{~Gb} / \mathrm{s}$

\begin{tabular}{cccc}
\hline $\begin{array}{c}\text { Modulation } \\
\text { Format }\end{array}$ & $\begin{array}{c}\text { Symbol Rate } \\
\text { [Gbaud] }\end{array}$ & Bits/symbol & $\begin{array}{c}\text { ROSNR [dB] } \\
\text { (@) } \begin{array}{c}\text { BER }=\mathbf{3 . 8} \times \mathbf{1 0}^{-\mathbf{3}} \\
\text { and Noise Bandwidth } \\
\mathbf{0 . 1} \mathbf{~ n m})\end{array}\end{array}$ \\
\hline IM-DD & 46 & 1 & 12.5 \\
DBPSK & 46 & 1 & 9.5 \\
DQPSK & 23 & 2 & 10.9 \\
DP-BPSK & 23 & 2 & 8.2 \\
DP-QPSK & 11.5 & 4 & 8.2 \\
DP-8QAM & 7.67 & 6 & 11.0 \\
DP-8PSK & 7.67 & 6 & 11.3 \\
DP-16QAM & 5.75 & 8 & 11.8 \\
\hline \hline
\end{tabular}

same bit rate, namely $46 \mathrm{~Gb} / \mathrm{s}$, and their respective constellations are illustrated in Fig. 1.

We use the required optical signal-to-noise ratio (ROSNR) as a metric of performance comparison. ROSNR is the ratio of optical signal power to noise power in a $0.1 \mathrm{~nm}$ bandwidth at which a bit error rate (BER) of $3.8 \times 10^{-3}$ is detected. Forward error correction (FEC), capable of a corrected error rate of $10^{-16}$ at the aforementioned raw error rate, is available in commercial product [7]. The symbol rate determines both the amount of optical spectrum occupied and the required speed of the electrical components. For a given ROSNR, lower symbol rates are preferred because the resulting lower spectral occupancy makes the transmission more tolerant to the optical flexibility equipment, such as reconfigurable optical add drop multiplexers (ROADMs), and because the relaxed bandwidth requirements allow the use of readily available lower cost electro-optics and high-speed electronics.

Table I lists three single-polarization noncoherent transceivers: intensity modulation direct detection (IM-DD), differential binary phase-shift keying (DBPSK), and differential QPSK (DQPSK). The balance of the table deals with polarization multiplexed coherent transceivers for which, at the receiver, polarization recovery is a simple linear matrix operation. For a given baud value polarization multiplexing doubles the channel capacity.

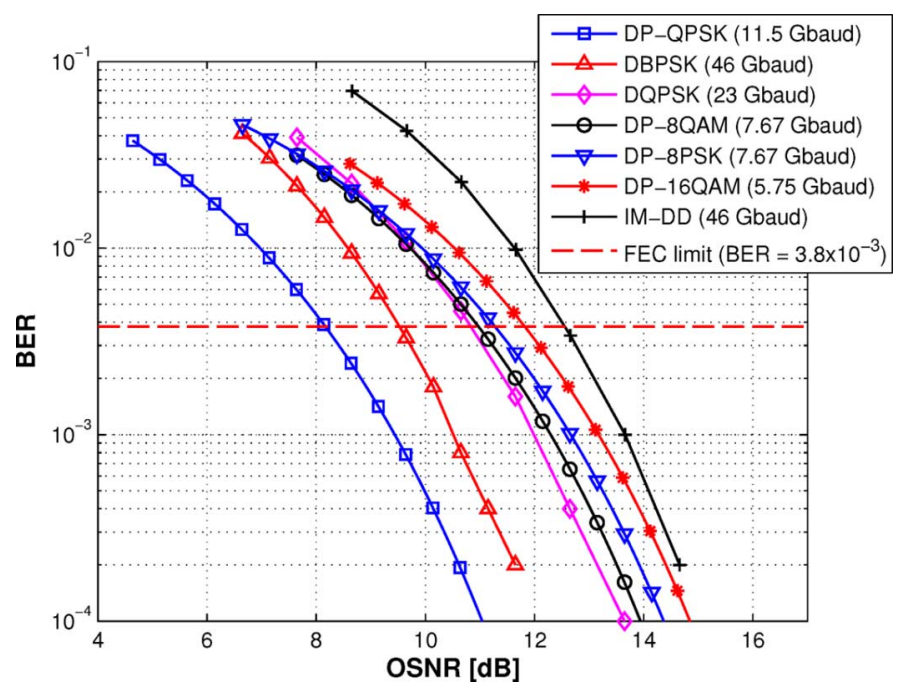

Fig. 2. BER versus OSNR for various modulation formats with $46 \mathrm{~Gb} / \mathrm{s}$ line rate. Resolution bandwidth $=0.1 \mathrm{~nm}$.

Fig. 2 shows the BER versus noise performance for a linear propagation of the selected modulation formats. The BER vs. OSNR curves for DP-QPSK (11.5 Gbaud), DP-8PSK (7.67 Gbaud) and DP-16QAM (5.75 Gbaud) are theoretical curves. An ideal square-root raised-cosine filter is assumed for the transmitter and receiver, respectively. The BER vs. OSNR curves for DBPSK (46 Gbaud) and DQPSK (23 Gbaud) were obtained through simulation, using an ideal balanced optical-delay-and-add interferometer and an ideal square-root raised-cosine filter at the transmitter and receiver, respectively. The BER vs. OSNR curve for IMDD (46 Gbaud) was also obtained through simulation using an ideal raised-cosine filter at the receiver before the PIN detector and a brick-wall filter (at twice the signal bandwidth) after the PIN detector. In the simulations, the rest of the transmitter and receiver are also assumed to be ideal.

At a BER of $3.8 \times 10^{-3}$, DP-BPSK and DP-QPSK have an ROSNR which is $1.3 \mathrm{~dB}$ lower than DBPSK and at least $2.7 \mathrm{~dB}$ lower than all other candidates. IM-DD and DBPSK have the highest baud values, namely 46 Gbaud, and require high-bandwidth components. At 5.75 Gbaud, DP-16QAM has the lowest baud value. However, its performance is inferior to DP-QPSK by 3.6 dB. DP-QPSK has a baud value of 11.5 Gbaud and, consequently, can be implemented with readily available $10-\mathrm{GHz}$ electro-optic components. By measure of ROSNR and baud value to achieve $46 \mathrm{~Gb} / \mathrm{s}$, DP-QPSK is, arguably, the best of listed in Table I.

\section{PRACTICAl ImPlementation}

\section{A. Architecture}

Fig. 3 shows a high-level block diagram for one sub-carrier of a coherent modem. Tributary signals, such as 10, 40, or $100 \mathrm{~Gb} / \mathrm{s}$ standard formats, are multiplexed and pre-coded with FEC having seven percent overhead. These bits are then multiplexed to a baud value of 11.5 Gbaud or 14 Gbaud. Four data streams modulate the light from a tunable laser, encoding in-phase and quadrature signals on $\mathrm{X}$ and $\mathrm{Y}$ polarizations. Thus, four data streams are combined and imposed on a single optical signal. Fig. 4 shows the measured optical spectra of the $40 \mathrm{~Gb} / \mathrm{s}$ 


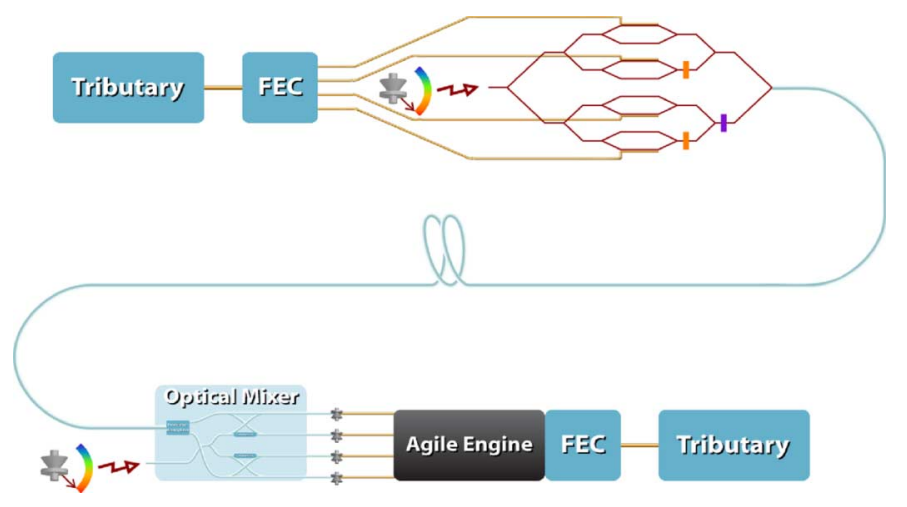

Fig. 3. Top level block diagram of coherent modem.

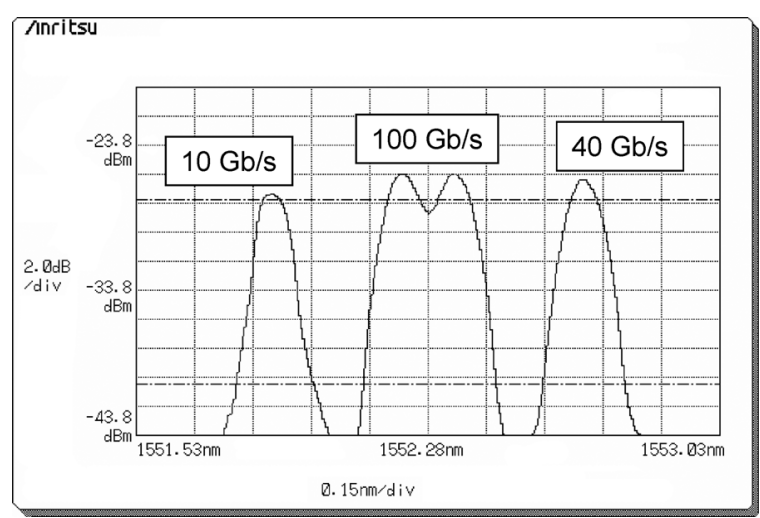

Fig. 4. Measured optical spectra for $10 \mathrm{~Gb} / \mathrm{s}$ IM-DD, $40 \mathrm{~Gb} / \mathrm{s}$ DP-QPSK, and $100 \mathrm{~Gb} / \mathrm{s}$ dual-carrier DP-QPSK. Each spectrum is centered in a $50-\mathrm{GHz}$ channel on the ITU grid. Resolution bandwidth $=0.07 \mathrm{~nm}$.

DP-QPSK, the dual-carrier $100 \mathrm{~Gb} / \mathrm{s}$ DP-QPSK, and for comparison, a $10 \mathrm{~Gb} / \mathrm{s} \mathrm{IM-DD} \mathrm{signal.}$

The transmission link is, typically, a cascade of fiber, amplifiers, filters, and ROADMs. The optical signal is mixed at the receiver with a laser which serves as a local oscillator. Fig. 5 shows how the signal and local oscillator are combined. The signal is first split along orthogonal polarization directions by means of a polarization beam splitter which serves as a local polarization reference. Each signal polarization is further split and combined with each of two mutually orthogonal phases of co-polarized light from the local oscillator. This is accomplished using splitters, phase shifters and combiners to deliver four orthogonal optical signals to the four photo-diodes. The local oscillator has to be swept so that its frequency is within a few hundred $\mathrm{MHz}$ of the transmitter laser frequency for acquisition in the digital signal processing (DSP) engine. The four electrical signal dimensions (two phase and two polarization dimensions) are then a complete set representing the optical signal which can be further demodulated. Of course, four anti-aliasing filters with proper bandwidth have to be implemented before the analog-to-digital converters (ADCs) to minimize aliasing noise. Under normal conditions the received signal's polarization orientation rotates with changes in the propagation path, the signal phase orientation rotates with the phase noise and the frequency difference between the transmit laser and the local oscillator, the symbol clock phase is not known, and the symbols have been severely distorted by propagation effects. Amplified spontaneous emission from the amplifiers also adds noise.

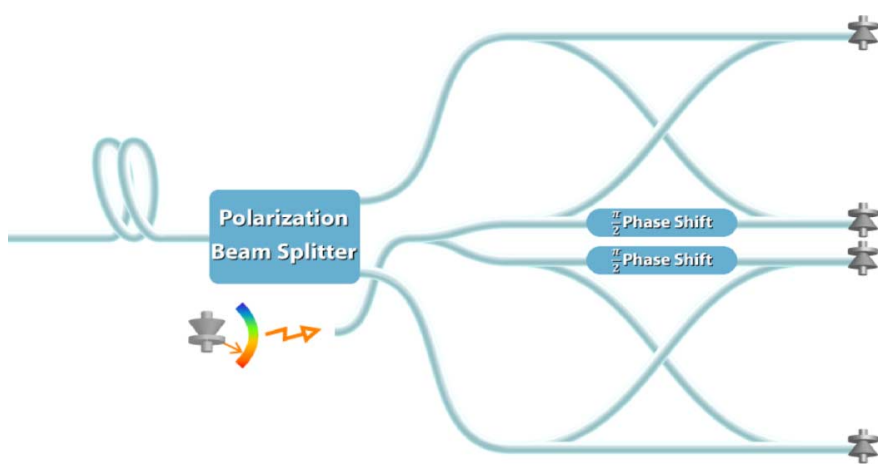

Fig. 5. Detail of coherent modem receiver optical front end.

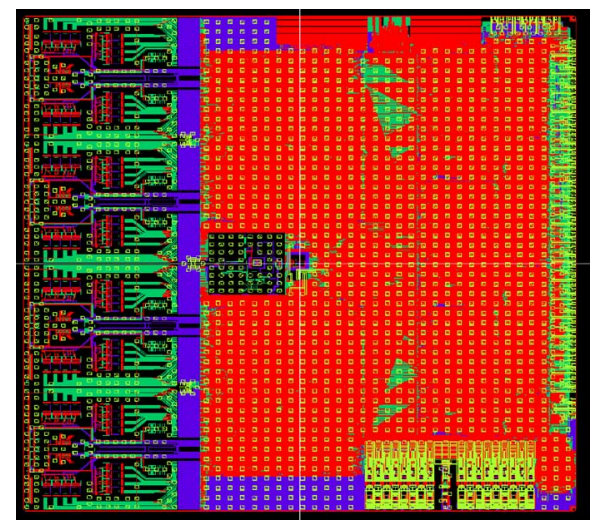

Fig. 6. Photograph of Agile Engine CMOS ASIC layout.

It is the task of the "Agile Engine" (i.e., receive analog-to-digital converters and digital signal processing functions) in Fig. 3 to recover the transmitted bits from the received signal. After amplification and automatic gain control, analog signals are converted to 6-bit digital values, sampled every half symbol interval $(\mathrm{T} / 2)$ to prevent aliasing. A photograph of the Agile Engine's 20-million gate CMOS ASIC layout is shown in Fig. 6. The four analog-to-digital converters are the dark blocks on left side. The central black rectangle is the voltage controlled oscillator that determines the sampling times. The red region contains the digital gates which execute 12 trillion integer operations per second to implement linear and nonlinear filtering that inverts the propagation effects and recovers the candidate data bits. These methods are described in later sections. The ASIC is manufactured in 90-nm commercial CMOS technology, and is approximately $12 \mathrm{~mm}$ by $16 \mathrm{~mm}$ in size.

This ASIC can compensate $>50000 \mathrm{ps} / \mathrm{nm}$ of chromatic dispersion and more than 100 ps of peak differential group delay due to PMD. It dissipates a total of $21 \mathrm{~W}$ of power. Because of the optical and processing noises, some of the candidate bits decoded from the optical signal will be in error. The FEC ASIC, which follows the Agile Engine ASIC in the receive path, corrects those errors, to a theoretical probability of a bit remaining in error of less than $10^{-20}$. (Long term stable tests with zero post-FEC errors have only been able to verify this theoretical performance down to the $10^{-16}$ level.) The bit streams are demultiplexed, producing exact copies of the original tributaries. 
TABLE II

POWER DISSIPATION COMPARISON FOR DISPERSION COMPENSATION: OPTICS VERSUS DSP

\begin{tabular}{lcccc}
\hline \hline & $\begin{array}{c}\text { Number } \\
\text { of } \\
\text { Channels }\end{array}$ & \multicolumn{2}{c}{$\begin{array}{c}\text { Dispersion- } \\
\text { Managed System } \\
\text { (Optical } \\
\text { Compensation) }\end{array}$} & $\begin{array}{c}\text { Non-Dispersion- } \\
\text { Managed System } \\
\text { (Electronic } \\
\text { Compensation) }\end{array}$ \\
\hline & & $\begin{array}{c}\text { High- } \\
\text { Power } \\
\text { Pump } \\
\text { [W] }\end{array}$ & $\begin{array}{c}\text { Additional } \\
\text { Pump }\end{array}$ & [W] \\
\hline $\begin{array}{c}\text { Power } \\
\text { dissipation } \\
\text { per EDFA }\end{array}$ & 1 & 5 & {$[\mathrm{~W}]$} \\
\hline $\begin{array}{l}\text { Total power } \\
\text { dissipation }\end{array}$ & 88 & 0.23 & 1.1 & 0 \\
\hline $\begin{array}{c}\text { Power } \\
\text { dissipation } \\
\text { per channel }\end{array}$ & 10 & 2 & 10 & 4.6 \\
\hline \hline
\end{tabular}

\section{B. Power Dissipation: Optics versus DSP}

The heat dissipation for the DSP section in the Agile Engine ASIC is approximately $10 \mathrm{~W}$ for the $46 \mathrm{~Gb} / \mathrm{s}$ DP-QPSK application. Of this, $4.6 \mathrm{~W}$ are generated by digital compensation for optical dispersion.

It is worth noting that the power consumed by digital compensation will fall as a result of the evolution of the semiconductor processes used to realize the required DSP.

Operation costs are, arguably, offset by some of the benefits of digital dispersion compensation. These include elimination of optical compensators and associated optical amplification, reduction of inter-channel nonlinear crosstalk (which will be described in Section V) and the near elimination of residual dispersion. Equipment reduction and performance improvement translate into lower network and operations cost.

Alternately, we can compare the heat dissipations associated with digital and optical dispersion compensations. We consider a unidirectional dispersion-managed link which uses in-line dispersion compensating modules and the same link in the absence of optical dispersion compensation. Both contain 20 spans of G.652 fiber with $80 \mathrm{~km} / \mathrm{span}$ for a total link dispersion of about $50000 \mathrm{ps} / \mathrm{nm}$. The comparison is summarized in Table II. Heat dissipation is listed in watts per channel. Gain accommodation for compensator loss at each EDFA is modeled to be provided either by increasing the pump laser power at a cost of $1 \mathrm{~W}$ per line amp site or by adding another pump laser at a cost of $5 \mathrm{~W}$ per line amp site. These values were taken from pump laser datasheets from various vendors [8]. We note that if a separate amplifier card is engendered to provide the extra gain, the marginal heat would be larger than modeled here. A full-fill system with 88 channels (50-GHz grid spacing in the C-band) is tabulated alongside a 10-channel case.

The marginal heat dissipated per channel in the digital compensation case is fixed whereas in the optical dispersion compensation case it depends on the number of channels. The channel fill at which the marginal heat per channel is the same for digital and optical dispersion compensation lies in the range between 4 and 22 channels for a 20-span link depending on the marginal heat model used.

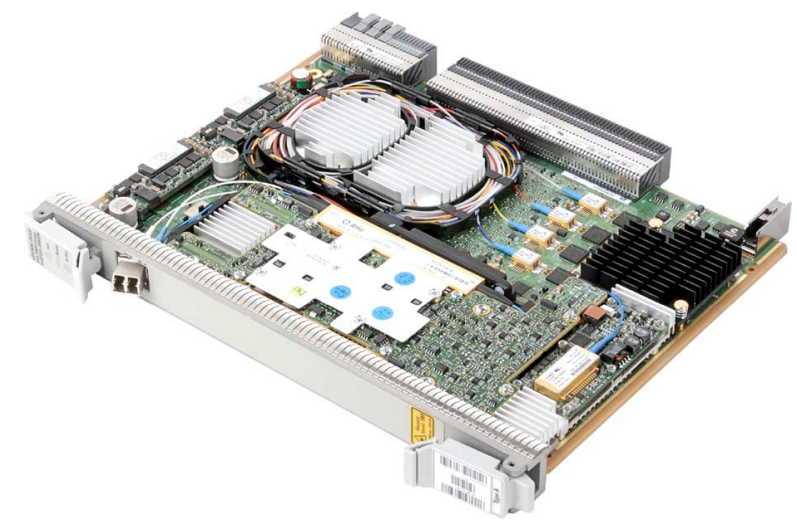

Fig. 7. Photograph of commercial $40 \mathrm{~Gb} / \mathrm{s}$ DP-QPSK coherent transceiver.

\section{Complexity}

Fig. 7 shows a photograph of a commercial $46 \mathrm{~Gb} / \mathrm{s}$ DP-QPSK coherent transceiver. The card measures approximately 8 in $\times 11$ in $\times 1$ in and includes multiplexing, FEC, and modem functions. The optical and analog functions, and their associated complexity, have been streamlined by the use of custom CMOS ASICs. These perform fast polarization recovery thereby displacing fast, variable delay polarization tracking optics for the polarization demultiplexing and PMD compensation. Similarly, the ASICs replace the function of tunable optical chromatic dispersion compensation. Digital compensation for the imperfections in the coherent mixer (90-degree optical hybrid) and in the low bandwidth electrical gain stages allow low cost analog components to be used. A block diagram showing the main building blocks of the Agile Engine ASIC in Fig. 3 is given in Fig. 8. The same tunable laser is used as the transmit source and as the local oscillator, to save the cost, heat, and space of a second laser.

\section{LinEAR PERFormance}

\section{A. Laser Phase Noise}

The laser phase noise is characterized by the linewidth parameter in $\mathrm{Hz}(L W)$. The phase noise can be quantified as the phase variance $\left(\sigma^{2}\right)$, which is proportional to an observation time $(\Delta t)$ and the linewidth, as follows [9]:

$$
\sigma^{2}=2 \pi(L W) \Delta t
$$

In practice, it is useful to relate the observation time to the symbol (baud) interval. Laser's linewidths have improved significantly in recent years such that DFB lasers with linewidths of 1 to $3 \mathrm{MHz}$ and external cavity lasers with linewidths of 10 to $100 \mathrm{kHz}$ are commercially available. The total phase noise consists of the linewidth contributions from transmitter and receiver lasers, as well as nonlinear phase noise and amplified spontaneous emission. Table III illustrates that laser phase noise is less of an issue for higher baud values since the corresponding phase variance per symbol is less. For example, a 28 Gbaud system is able to tolerate more than twice the laser linewidth as compared to a 10 Gbaud system, using the same design.

DP-QPSK has a phase margin of 45 degrees; so for example, a Gaussian standard deviation exceeding 15 degrees would result 


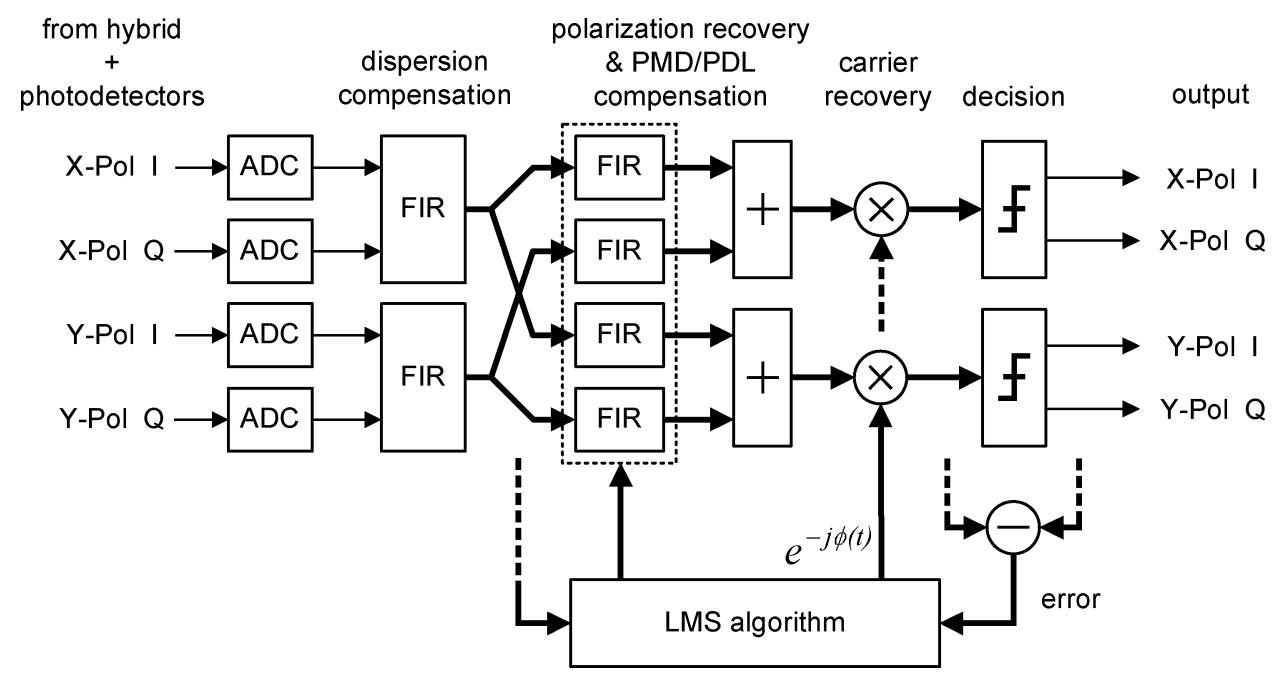

Fig. 8. Block diagram of the digital signal processing building blocks composing the ASIC of the Agile Engine on the $40 \mathrm{~Gb} / \mathrm{s}$ DP-QPSK coherent transceiver [10]. ADC: analog-to-digital converter, FIR: finite impulse response linear filter, LMS: least-mean square.

TABLE III

EFFECT OF LASER LINEWIDTH AND BAUd RATE ON SyMBOL PHASE DEVIATION

\begin{tabular}{lccc}
\hline $\begin{array}{c}\text { Total } \\
\text { Linewidth } \\
(\mathbf{T x}+\mathbf{R x})\end{array}$ & $\begin{array}{c}\text { Symbol Rate } \\
\text { [Gbaud] }\end{array}$ & $\begin{array}{c}\text { RMS Deviation } \\
\text { [degree/symbol] }\end{array}$ & $\begin{array}{c}\text { Symbols to } \\
\mathbf{1 5} \text { degrees }\end{array}$ \\
\hline $2 \times 1 \mathrm{MHz}$ & 10 & 2.03 & 55 \\
\hline $2 \times 100 \mathrm{kHz}$ & 28 & 1.21 & 156 \\
\hline \hline
\end{tabular}

in substantial errors even in the absence of other noise sources. The time duration, in number of symbols, until this condition is reached is shown in Table III. To prevent this issue in a coherent receiver it is necessary to track the laser phase using a carrier recovery circuit. First, the phase is detected, and then the result is filtered. The filter bandwidth is selected to optimally track the joint laser phase deviation in the presence of the optical noise.

The main challenge in phase detection is the data modulation that is present. A decision directed phase detector requires a current phase estimate, such as from a very fast second-order phase-locked feedback loop [11], [12]. Fourth-power and other symbol-independent phase detectors allow the use of a feedforward circuit [13]. For dual-polarization signals, one can choose to average carrier phase estimates from the two polarizations, or use separate carrier phase estimates for each polarization. The choice depends upon the degree of correlation between the phases of the two polarizations.

Fig. 9 compares the performance of a noncausal feedforward carrier recovery loop against a causal feedback loop. The tracking error in degree RMS is plotted against loop bandwidth for a baud value of 11.5 GBaud. The DP-QPSK signal is loaded with amplified spontaneous emission noise to achieve a BER of $3.8 \times 10^{-3}$, and phase detection is polarization averaged (i.e., both polarizations are used). For a combined transmitter/receiver linewidth of $2 \mathrm{MHz}$, the optimum bandwidth is about $400 \mathrm{MHz}$.

It is very advantageous to be able to tolerate the phase noise from inexpensive DFB lasers. The selection of either a feedfor-

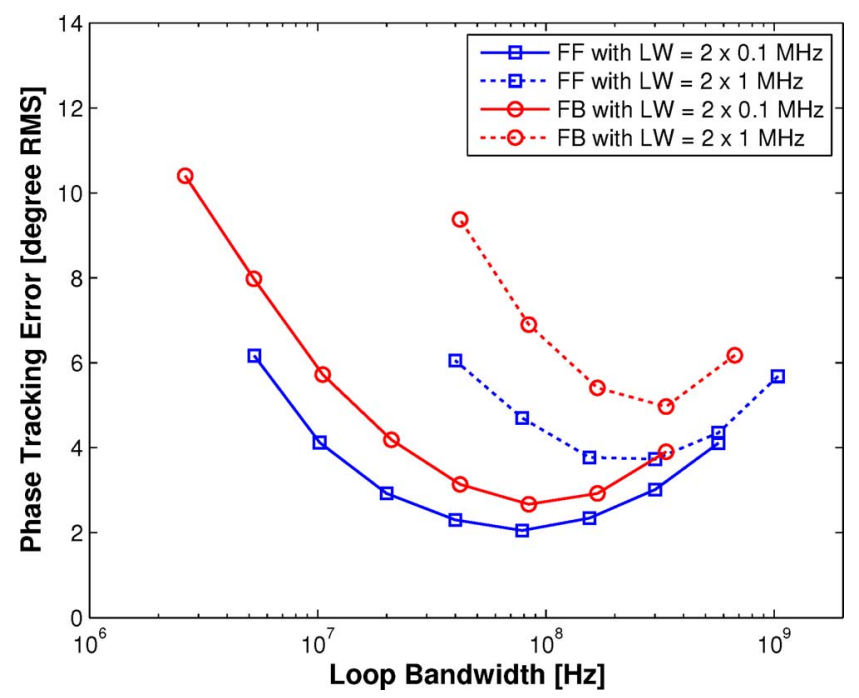

Fig. 9. Performance comparison of feedforward and feedback carrier recovery methods for DP-QPSK modulation format at 11.5 Gbaud and $\mathrm{BER}=3.8 \times 10^{-3}$. Both polarizations are used. FF: feedforward; FB: feedback; LW: linewidth.

ward or feedback approach to achieve this requires a detailed evaluation of the implementation constraints. Loop delay will limit the maximum feedback loop bandwidth that can be made stable. With careful design in current CMOS technology, stability can be achieved in a feedback loop with a $1 \mathrm{GHz}$ loop bandwidth. There is no such delay constraint in a feedforward design. A feedforward circuit can use memory to implement a noncausal filter, but requires significantly more gates. Thus, feedforward designs can be more easily made tolerant to large linewidth lasers, at the price of increased heat dissipation and circuit complexity relative to a feedback implementation.

\section{B. Dispersion Compensation}

Dispersion is a linear operation on the electrical field (E-field) and so its effects can be undone by linear filtering. The transfer 


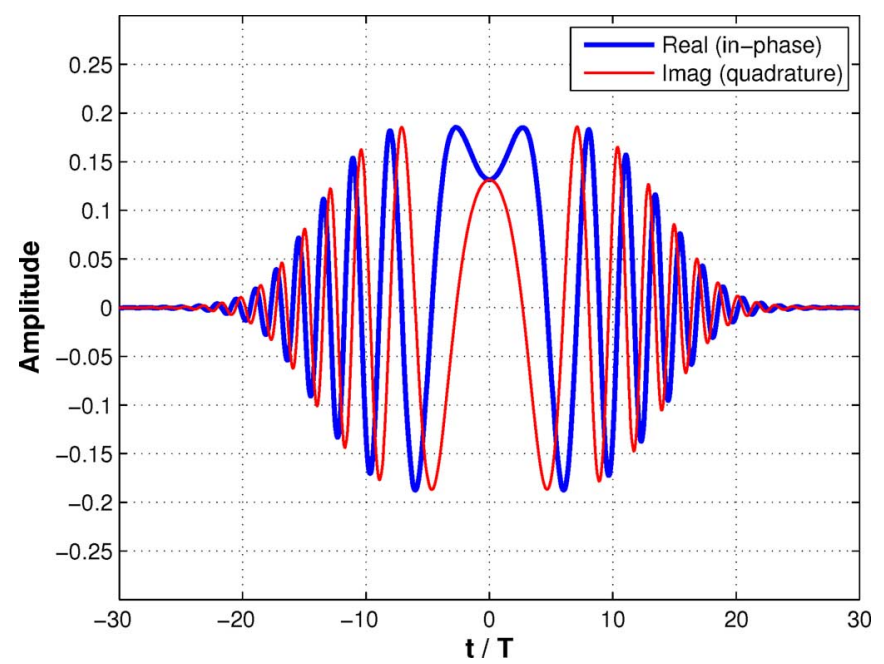

Fig. 10. Example of impulse response for dispersion $=27200 \mathrm{ps} / \mathrm{nm}$ with an ideal raised-cosine filter (roll-off factor $=0.5$ ).

function of the fiber with chromatic dispersion is modeled as [14]

$$
H_{\text {disp }}(f)=\exp \left(-\frac{j \beta_{2} \omega^{2} L}{2}\right)
$$

where $\beta_{2}$ is the group-velocity dispersion (GVD) parameter in $\mathrm{ps}^{2} / \mathrm{km}, \omega$ is the angular frequency of the signal in $\mathrm{rad} / \mathrm{s}$, and $L$ is the length of the fiber in $\mathrm{km}$. $\beta_{2}$ is equal to $-D \lambda^{2} /(2 \pi c)$, where $D$ is the dispersion parameter of the fiber in $\mathrm{ps} /(\mathrm{nm}-\mathrm{km})$, $\lambda$ is the wavelength of the laser source in $\mathrm{nm}$, and $c$ is the speed of light in vacuum [15]. Note that $H_{\text {disp }}(f)$ is an all-pass function with a phase which is proportional to the square of frequency. To see the effect of dispersion, it is necessary to consider the signal bandwidth. With an ideal raised-cosine filter for a 10 Gbaud signal the impulse response due to $27200 \mathrm{ps} / \mathrm{nm}$ of dispersion is shown in Fig. 10. This represents $1600 \mathrm{~km}$ of G.652 fiber having a dispersion characteristic of $17 \mathrm{ps} / \mathrm{nm} / \mathrm{km}$.

The illustrated pulse response spans about 45 symbols, as does its conjugate which is the dispersion compensating impulse response. Therefore, a linear finite impulse response (FIR) filter, with $90 \mathrm{~T} / 2$-spaced taps is able to completely undo the dispersion, i.e., to do post-dispersion compensation. For a given baud value, the required number of $\mathrm{T} / 2$ filter taps depends linearly on the amount of chromatic dispersion to be compensated. Thus, a 152-tap linear filter with $\mathrm{T} / 2$ spacing can compensate up to $\pm 50000 \mathrm{ps} / \mathrm{nm}$ of dispersion at $10 \mathrm{Gbaud}$ with no measurable performance penalty. Moreover, this filter can also correct some of the imperfections in the analog hardware frequency responses.

Fig. 11 shows single-channel propagation performance of a commercial coherent $40-\mathrm{Gb} / \mathrm{s}$ transceiver modem on 43 spans each consisting of $80 \mathrm{~km}$ of G.652 fiber, without any optical dispersion compensation. In this experiment a single channel was used at $1546.92 \mathrm{~nm}$ and launch power was set to $-4 \mathrm{dBm}$. The mean PDL is $1.5 \mathrm{~dB}$. The measurements were done after each span between 23 spans $(\sim 31000 \mathrm{ps} / \mathrm{nm})$ and 43 spans (> $55000 \mathrm{ps} / \mathrm{nm})$. The filter taps are chosen by inverting (2).

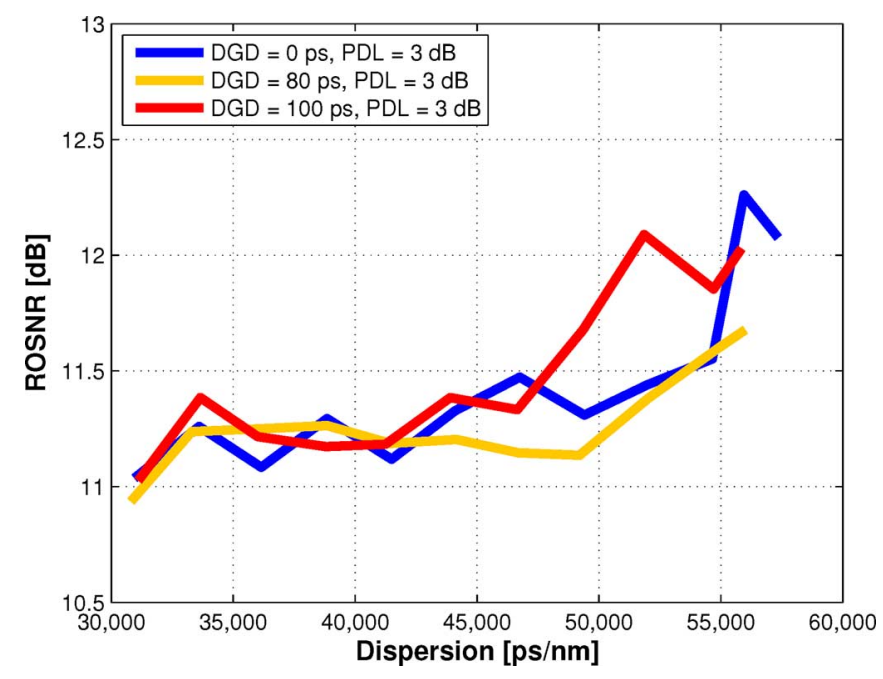

Fig. 11. Required OSNR as a function of link dispersion and PMD for singlechannel propagation on G.652 fiber. Wavelength is set to $1546.92 \mathrm{~nm}$. Launch power is set to $-4 \mathrm{dBm}$. Resolution bandwidth $=0.1 \mathrm{~nm}$.

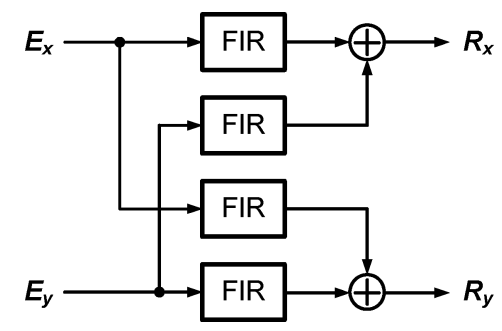

Fig. 12. Multiple-input multiple-output equalization structure. FIR: finite impulse response linear filter.

Note the increased slope after $50000 \mathrm{ps} / \mathrm{nm}$, which is due to the 152 effective filter taps no longer being sufficient to completely compensate the dispersion. The system can compensate $\pm 80000 \mathrm{ps} / \mathrm{nm}$ of total chromatic dispersion, with a $2 \mathrm{~dB}$ penalty.

\section{Polarization Recovery}

Polarization, PMD, and PDL of the fiber channel can be described as a frequency dependent $2 \times 2$ network of filter elements:

$$
\left[\begin{array}{l}
R_{x} \\
R_{y}
\end{array}\right]=\left[\begin{array}{ll}
H_{x x}(\omega) & H_{y x}(\omega) \\
H_{x y}(\omega) & H_{y y}(\omega)
\end{array}\right]\left[\begin{array}{l}
E_{x} \\
E_{y}
\end{array}\right]
$$

where $R, H$, and $E$ are received $\mathrm{X}$-pol/Y-pol signals, $2 \times 2$ complex channel responses, and transmitted X-pol/Y-pol signals, respectively. With finite PDL, the channel matrix is invertible for each frequency component of interest. This leads to a multiple-input multiple-output equalization structure with four linear filters (Fig. 12), which corresponds to the center section of Fig. 8 [16]. However, inversion of distributed PDL in the presence of distributed noise causes OSNR penalty. Interleaving of bits across polarization tributaries after equalization can significantly reduce this penalty [17].

Nearly complete equalization is easily achieved given that the time spans of the linear filters are sufficiently long to cover the inverse of the polarization-dependent channel response. PMD tolerance of a commercially available modem [18] is 


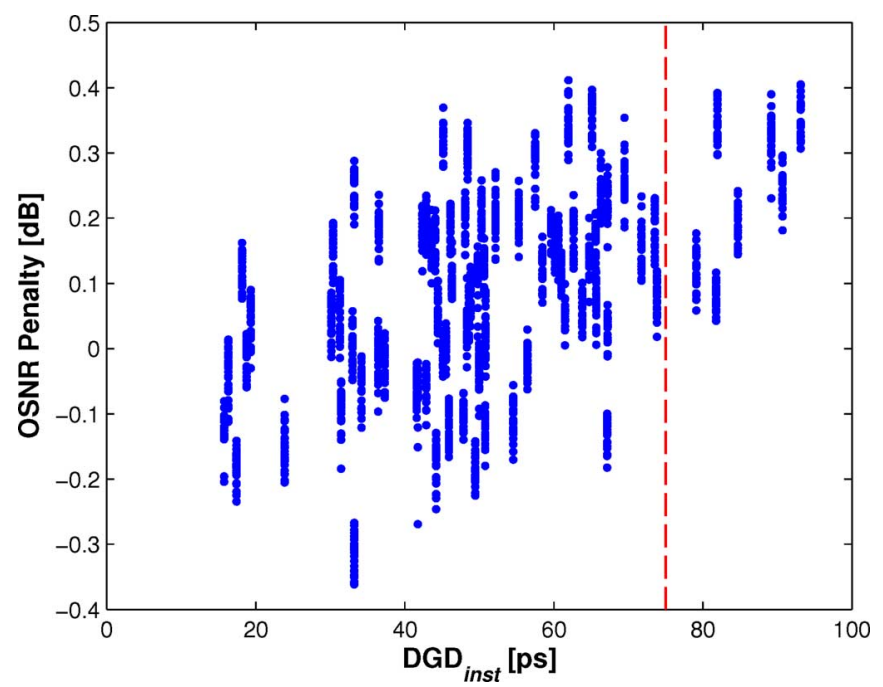

Fig. 13. DGD tolerance of commercial DP-QPSK modem (single channel) relative to back-to-back. Dashed line: DGD tolerance specification of commercial coherent modem of Fig. 7.

measured in [19], and the results are shown in Fig. 13. The DP-QPSK modem was subjected to a transmission path composed of distributed high-PMD fibers with near-Maxwellian PMD statistics for differential group delay (DGD) as well as all higher orders, as expected for a long mode-coupled fiber link. Polarization states were randomly chosen to generate a variety of DGD values up to 100 ps (peak) in a single channel experiment and to $130 \mathrm{ps}$ in a WDM experiment. (See [19] for a detailed discussion of the experiments and WDM measurements at very high DGD values.) Fig. 13 shows DGD tolerance for single channel measurements. Each DGD value is obtained by a random choice of polarization states. The PMD vector is independently measured by the Mueller matrix method in order to determine the DGD value at a given wavelength. OSNR penalty is plotted against instantaneous DGD in picoseconds relative to back-to-back. For each DGD value the signal was launched at 26 different polarization states, well distributed on the Poincaré sphere. Small OSNR penalties are observed across large range of DGD values. Also included on the graph is the 75 ps peak DGD tolerance specification of the commercial coherent modem (vertical dashed line).

The adaptation of the filter coefficients can be done by known algorithms such as RLS, LMS, and CMA [20]. The RLS algorithm has the fastest convergence, but also the most complexity. LMS and CMA algorithms have slower convergence, but are easier to implement and can be made sufficiently fast to equalize polarization responses in the kilohertz range [21]. The adaptation of the filter coefficients must be hardware driven in order to efficiently track fast polarization transients.

Polarization rotation rates of up to $40 \mathrm{kHz}$ can be excited by mechanically shocking the coils of fiber in dispersion compensating modules [22]. Mechanical shock on the chassis generates stress in the fiber which results in these large and fast polarization transients. To characterize this effect, an unmodulated optical source was coupled into a dispersion compensating module (DCM) and the Stokes parameters were measured at the output (Fig. 14). A steel ball $12.7 \mathrm{~mm}$ in diameter was dropped from

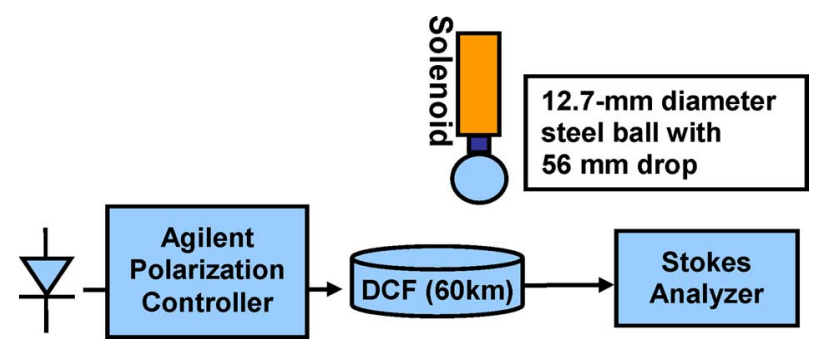

Fig. 14. Setup for analyzing fast polarization transients due to mechanical shocks on a dispersion compensating module.

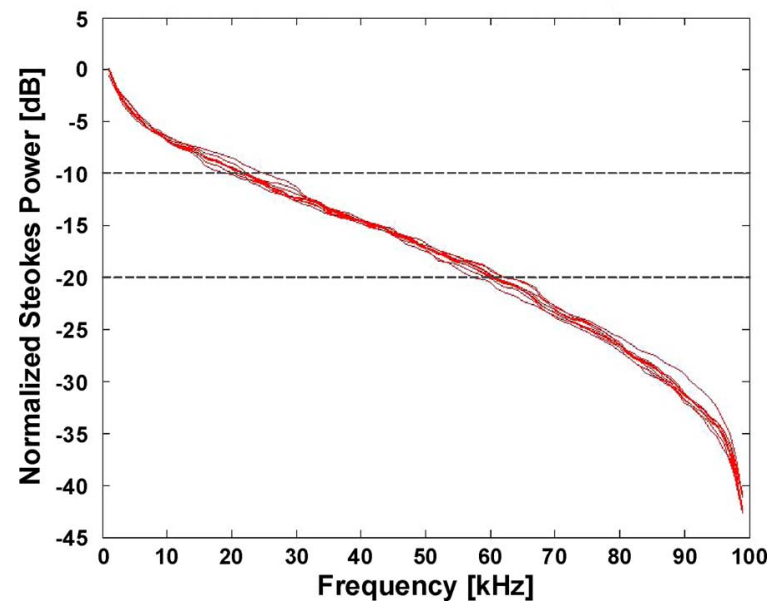

Fig. 15. Polarization rate of change induced by mechanical shocking of DCM.

a $56 \mathrm{~mm}$ height onto a fixed position on the chassis, and the Stokes parameters were extracted at high speed. Stokes power density, $P(f)$, is defined as follows:

$$
P(f)=\sum_{k}\left|S_{k}(f)\right|^{2}
$$

$S_{k}(f)$ is the Fourier transform of the $k$ th Stokes parameter. From results processed in this way, Fig. 15 shows that there was modulation energy $10 \mathrm{~dB}$ down from maximum at $20 \mathrm{kHz}$ and $20 \mathrm{~dB}$ down at $60 \mathrm{kHz}$.

The hardware adaptation of the equalization coefficients can easily deal with this type of transient, owing to the high data rate and fast update that can be achieved in the ASIC. To demonstrate this, the dispersion compensation module was placed between two first-order DGD elements (each set at $30 \mathrm{ps}$ ), and the steel ball was dropped 20 times for each OSNR value (Fig. 16). During each drop, error counts were collected every millisecond over a 20 -second duration covering the relaxation time of the mechanical resonance. The worst case BER from the 20 repeats of 20-second recording is reported as the BER for each OSNR value. The results are shown in Fig. 17 and show $0.5 \mathrm{~dB}$ of OSNR penalty for such extreme polarization transients (open square symbols). The transient results reported in Fig. 17 are comprised of measurements for which no post-FEC errors were detected ( 20 drops per OSNR value times five OSNR values for a total of 100 drops for this experiment).

The equalization tap values can also be used for monitoring chromatic dispersion, states of polarization, and PMD of the fiber channel. Time-varying PMD statistics can be used for link 


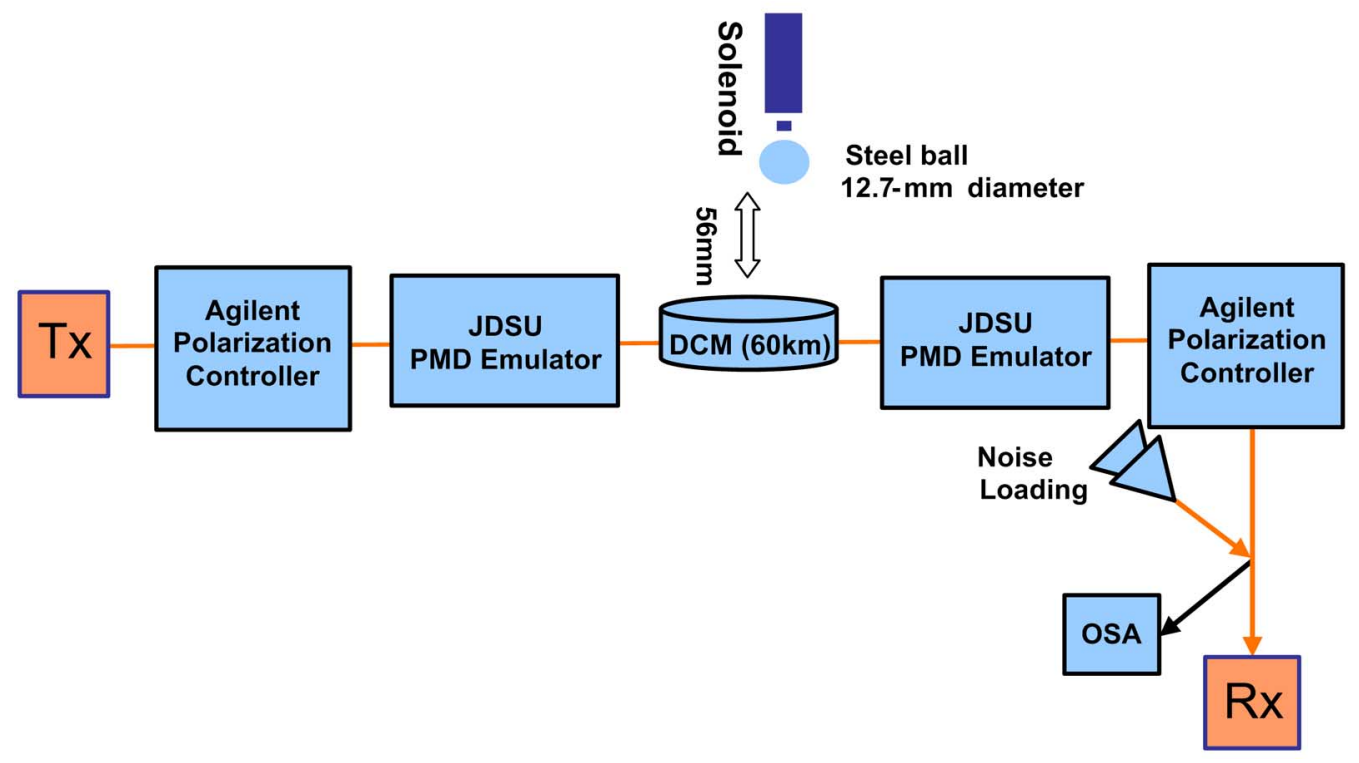

Fig. 16. Setup for PMD transient performance measurements. OSA: optical spectrum analyzer.

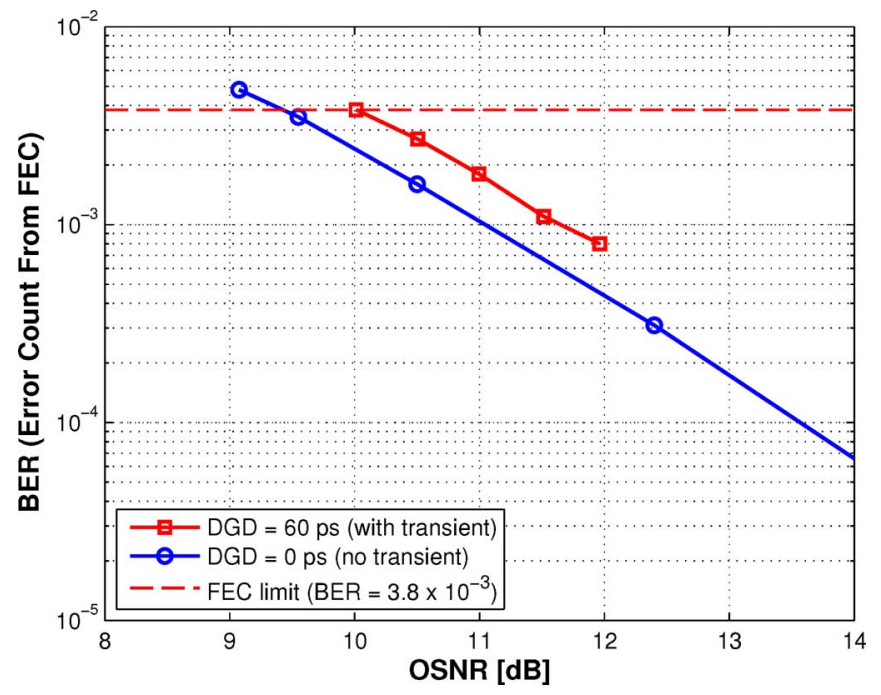

Fig. 17. Polarization transient performance. Resolution bandwidth $=0.1 \mathrm{~nm}$.

characterization and fault isolation, and can be used proactively to address impairments. Accuracies of $32 \mathrm{ps} / \mathrm{nm}$ for chromatic dispersion and 12 ps (at 95\% confidence) for DGD values ranging from 10 to $120 \mathrm{ps}$ were measured on a commercial $40-\mathrm{Gb} / \mathrm{s}$ coherent modem [23].

\section{Filter Tolerance}

Another important system parameter is the tolerance to frequency misalignment between a transmitter and the filters and optical switches along the optical path. This is especially challenging when implementing 40-Gb/s WDM transmission with a spacing of $50-\mathrm{GHz}$ between channels. The transceiver's tolerance to such frequency offsets was measured using a 50-GHz wavelength selective switch (WSS) in a recirculating loop (Fig. 18). The recirculating loop allows the emulation of many WSS without having to procure a large number of them. The full-width at half-maximum of the power transmission spectrum of the WSS is $44 \mathrm{GHz}$. This experiment used a benchtop lab transceiver with a real-time sampling oscilloscope and post-processing on the coherent receiver side. The same signal processing algorithms as used in the commercial $40 \mathrm{~Gb} / \mathrm{s}$ coherent modem were adapted for processing the burst that circulated through the loop. The back-to-back ROSNR of the lab transceiver was approximately $1.2 \mathrm{~dB}$ lower than a typical commercial unit.

The measured optical spectrum of the $40 \mathrm{~Gb} / \mathrm{s}$ DP-QPSK transmitter is given in Fig. 19 as well as the filter responses of a 50-GHz multiplexer/demultiplexer (mux/demux) pair and wavelength selective switch. The ROSNR was measured versus the frequency offset and is shown in Fig. 20. Maximum OSNR penalties of $1.5 \mathrm{~dB}$ are observed after 20 orbits for frequency offsets of up to $\pm 10 \mathrm{GHz}$. The worst case offset expected in a system is $\pm 5 \mathrm{GHz}$. Owing to the difference in back-to-back ROSNR a typical commercial unit is estimated to sustain an OSNR penalty of $2.1 \mathrm{~dB}$ after traversing 20 WSS.

\section{NONLINEAR PERFORMANCE}

We simulate the XPM action of interfering pump channels on a $46 \mathrm{~Gb} / \mathrm{s}$ or $112 \mathrm{~Gb} / \mathrm{s}$ DP-QPSK probe channel using a pump intensity to probe phase transfer function approximation [24], [25]. The nonlinear phase imparted to the the probe channel is evaluated at zero net dispersion. This dispersion condition is prerequisite for detection in a phase modulated system.

Usually, the total field including inter-channel nonlinear effects is calculated by solving the nonlinear Schrödinger equation using split-step Fourier methods. For systems of commercial dimension, namely approximately 100 channels over 20 spans ( $80 \mathrm{~km}$ per span), this total field approach can be time consuming and does not easily offer insight into physical layer design. For example, the statistics of the effect of data patterns on the variance of the nonlinear phase are often inadequately explored by the short pattern lengths which are chosen in an effort to contain the calculation time. 


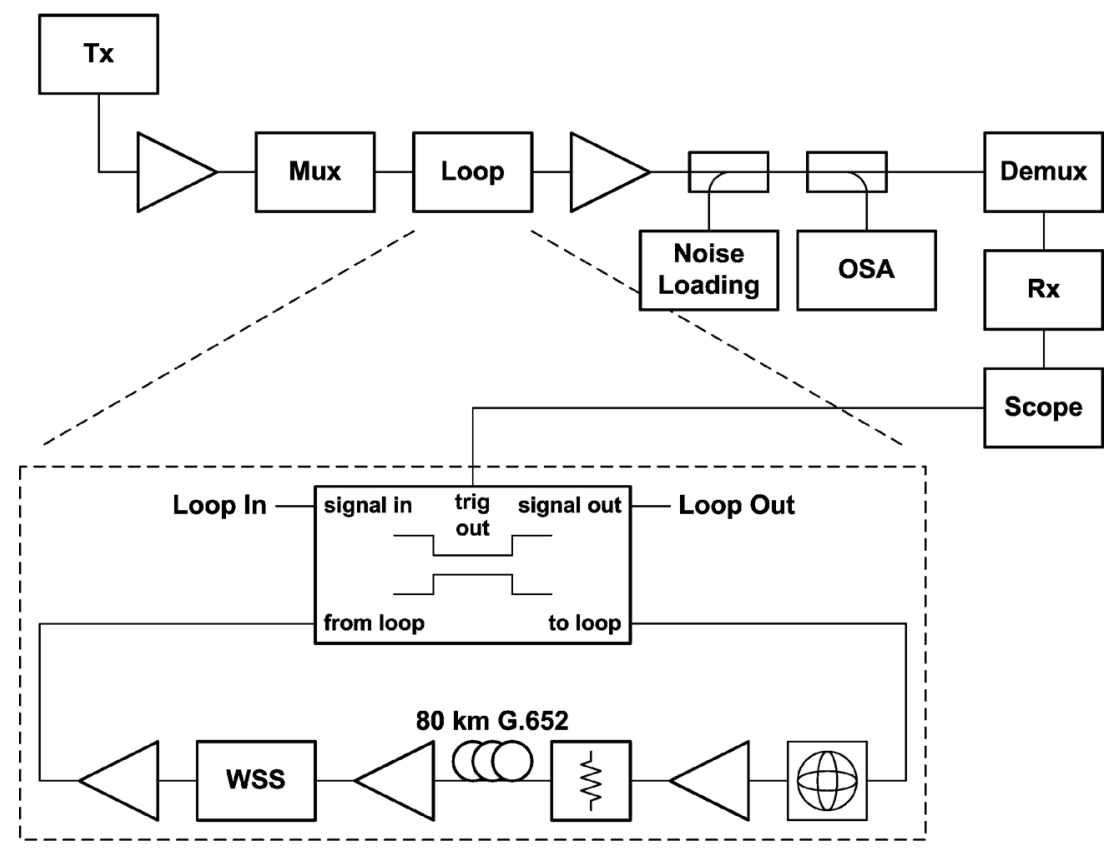

Fig. 18. Setup used for measuring filter tolerance using a recirculating loop.

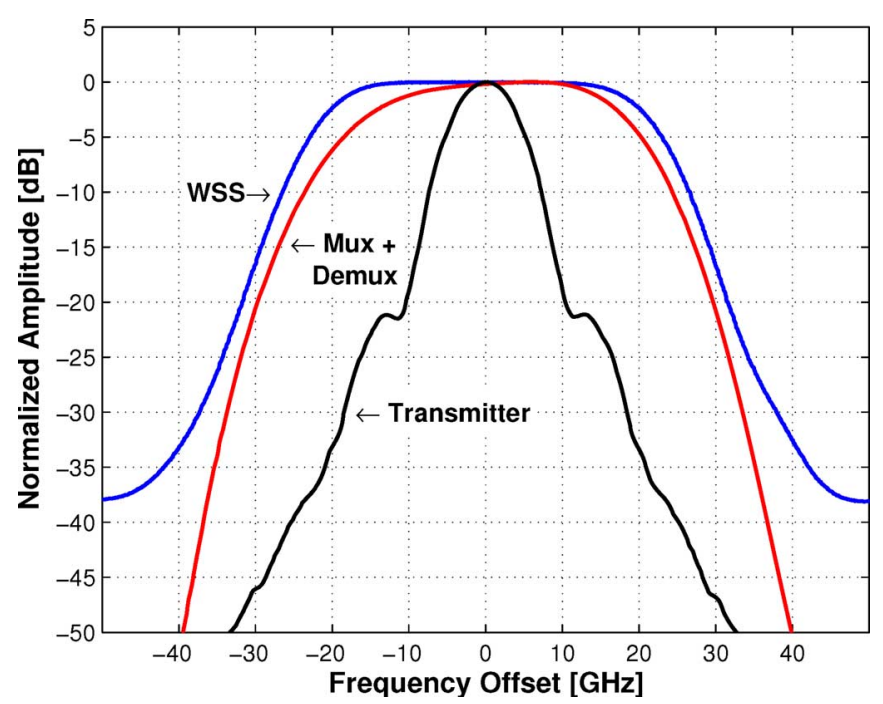

Fig. 19. Measured optical spectrum of $40 \mathrm{G}$ DP-QPSK transmitter and measured transmission responses of $50-\mathrm{GHz}$ mux/demux pair and WSS. Resolution bandwidth $=0.01 \mathrm{~nm}$.

From a first-order perturbation of the nonlinear propagation equation we get an approximate closed-form solution for the nonlinear phase which provides physical design insight. We neglect self-phase modulation (SPM) within pump channels and estimate their XPM effect on a probe channel. The original pump channel waveform, subject to linear propagation, can be used as the source of the phase perturbation. We further neglect subsequent SPM effects on these perturbations within the probe channel. Thus, the phase noise contribution from a WDM group of pump channels is the sum of contributions of individual pump channels. Since independently modulated WDM channels are statistically independent, the total power spectral density of the induced phase noise is the sum of the

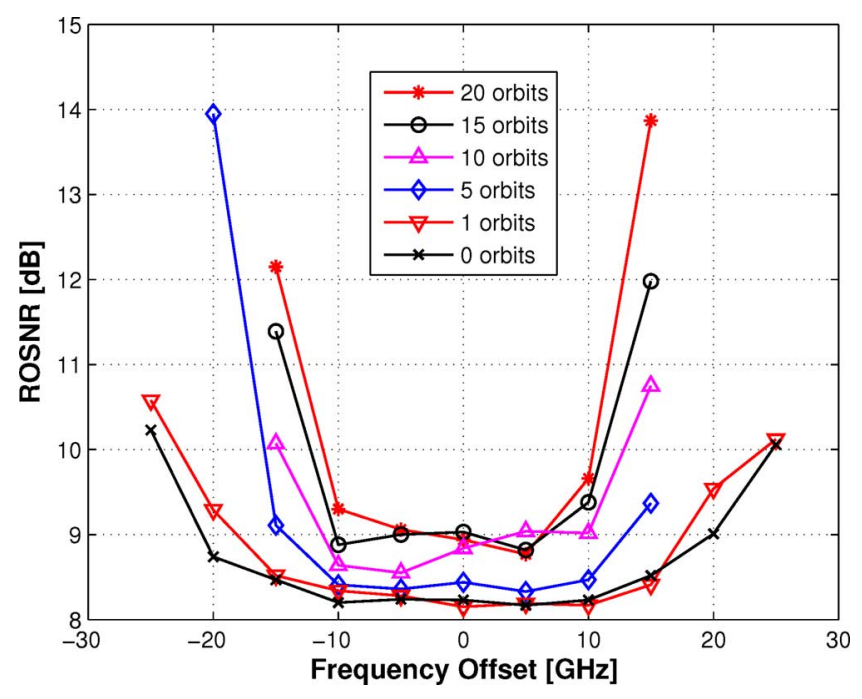

Fig. 20. Measured OSNR penalty vs. frequency offset between $40-\mathrm{Gb} / \mathrm{s}$ DP-QPSK transceiver and 50-GHz mux/demux and WSS filters. Resolution bandwidth $=0.1 \mathrm{~nm}$.

power spectral density due to each pump channel. This allows the analysis of a full WDM spectrum to be simplified by considering one interfering channel at a time.

In many wavelength division multiplexed (WDM) network applications it is desirable to support a mixture of different modulation formats travelling together on the same fiber. The transmission performance of these signals is influenced by nonlinear crosstalk between channels. An important part of this crosstalk is XPM. Phase modulated systems operate at zero net dispersion, where this sum includes all of the fibers, optical, and digital dispersive elements. With zero net dispersion, the amount of XPM imparted to a probe channel by a pump channel depends on the modulation format of the pump channel only. Conversely, the impact of XPM upon the transmission performance depends 
upon the modulation format of the probe channel only. Therefore, considering the simple pair-wise combinations of pump and probe formats is sufficient and the general WDM case is the power sum of these simple cases.

The effects of SPM and XPM depend strongly on the choice of optical dispersion map. The nonlinear field generated by SPM over an elemental section of fiber is proportional to the local instantaneous power. As a result of linear propagation dispersion accumulates and affects the local peak-to-average power ratio. For conventional modulation formats, such as those listed in Table I, the peak-to-average power ratio grows with the dispersion accumulated between the transmitter and a given elemental fiber section. Thus, SPM accumulated along the link length is greater when, in those regions of higher optical power (e.g., the outputs of line amplifiers), the net dispersion is large.

Another aspect is the degree of coherence, at the receiver, of nonlinear fields contributed by SPM from elemental fiber sections. In particular, if $B$ is the baud value, $D_{\text {net }}$ is the net dispersion per span, $\lambda$ is the channel wavelength, and $c$ is the speed of light, then define $k$ to be

$$
k=\frac{B^{2} \times D_{\text {net }} \times \lambda^{2}}{c} .
$$

When $k$ is close to an integer value there will be a higher degree of coherence among the nonlinear fields arriving at the receiver and so the power of their sum will be greater.

In the case of XPM, nonlinear phase modulation has a low-pass intensity-to-phase transfer function whose bandwidth decreases with local fiber dispersion. Effects related to high instantaneous peak-to-average power ratio, seen in SPM, are usually filtered out by this transfer function. Thus, XPM is lower in fibers with higher local dispersion.

Coherent addition of the XPM effects are strongest when the net dispersion per span is such that

$$
\frac{1}{B} \gg D_{\text {net }} \times \Delta \lambda
$$

where $\Delta \lambda$ is the channel separation. Under these conditions, the data alignment between channels substantially repeats at each span. This gives rise to higher XPM powers.

The relative strengths of XPM and SPM affecting $46 \mathrm{~Gb} / \mathrm{s}$ and $112 \mathrm{~Gb} / \mathrm{s}$ DP-QPSK channels on 20 spans of G.652 fiber are displayed in Fig. 21. The effect of $11.5 \mathrm{~Gb} / \mathrm{s} \mathrm{IM-DD} \mathrm{inter-}$ ference channels is also shown. The IM-DD pump model contains electronic pre-compensation for chromatic dispersion set to compensate for residual link dispersion. In this figure, the total angular standard deviation arising from XPM and SPM, obtained by repeated split-step Fourier solution to the nonlinear Schrödinger equation for pump-probe pairs with different frequency separations, is plotted versus the percentage of optical dispersion compensation per span. The nonlinear interaction between polarizations of the probe is label SPM for this discussion. 88 channels are spaced $50 \mathrm{GHz}$ apart, and the center channel is used as the probe. This channel transports either $46 \mathrm{~Gb} / \mathrm{s}$, $112 \mathrm{~Gb} / \mathrm{s}$ single-carrier, or $112 \mathrm{~Gb} / \mathrm{s}$ dual-carrier (CoFDM), all using DP-QPSK modulation format.

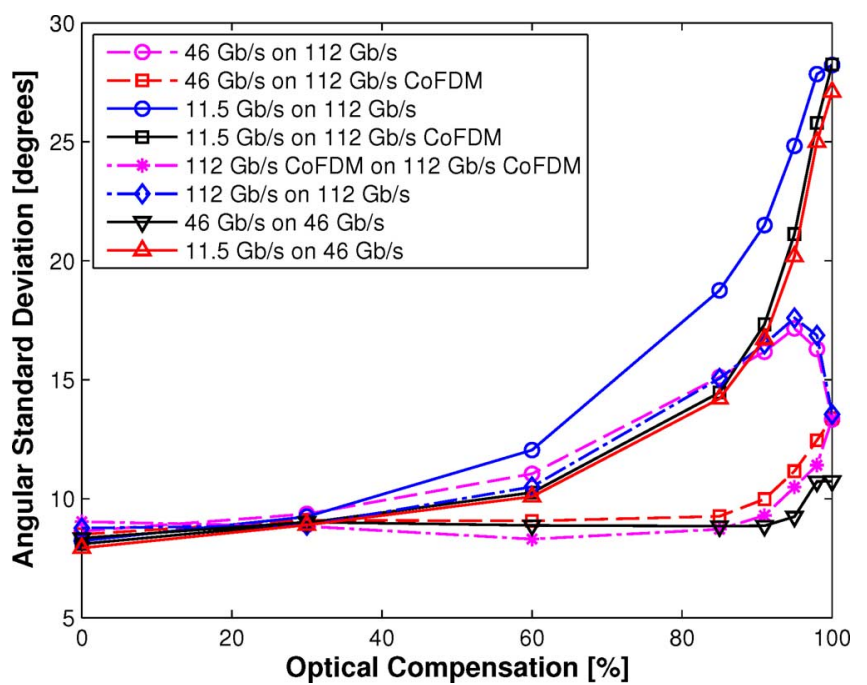

Fig. 21. Angular noise standard deviation, evaluated at zero net dispersion, versus percent optical compensation per span. The angular noise stems from SPM and XPM which is calculated to have accrued onto a central channel by propagation over a 20 -span $(1600 \mathrm{~km})$, G.652 fiber, 88-channel, WDM link. The spectral composition of each case is detailed in the legend where the first rate quoted refers to the interfering (pump) channels and the second rate refers to the central (probe) channel.

The $46 \mathrm{~Gb} / \mathrm{s}$ channels have an average power of $-0.5 \mathrm{dBm}$. The powers of the $11.5 \mathrm{~Gb} / \mathrm{s}$ and $112 \mathrm{~Gb} / \mathrm{s}$ channels are, respectively, $3 \mathrm{~dB}$ lower and higher to approximate optimum system BER performance due to noise. The angular standard deviation is seen to be approximately 8.5 degrees at $0 \%$ dispersion compensation per span which can provide adequate system margin for the DP-QPSK probes.

Fig. 21 shows that nonlinear phase accumulated during propagation increases with the percentage of optical dispersion compensation per span. With $11.5 \mathrm{~Gb} / \mathrm{s}$ pump wavelengths, the rise of angular noise at high percentages is the result of XPM. With single-carrier $112 \mathrm{~Gb} / \mathrm{s}$ probe signals the peaking of angular noise near $90 \%$ compensation is mostly due to SPM.

\section{COHERENT FREQUENCY SELECTION}

In a coherent optical receiver, the optical signal is mixed with the local oscillator to create electrical beat products after detection. The low-pass electrical filter applied to these beat products determines the portion of optical spectrum around the local oscillator frequency that is received. Much finer control of this optical spectrum shaping can be achieved using the baseband digital electrical filter than is feasible with optical filters.

Two carriers can be placed $20 \mathrm{GHz}$ apart, inside the spectrum of a single 50-GHz spaced ITU grid (Fig. 22), and coherent selection used at the receiver to distinguish them. When modulated with dual-polarization QPSK, these two carriers can transport $112 \mathrm{~Gb} / \mathrm{s}$. At a little more than two bit/s/Hz of spectral efficiency, a C-band amplified fiber connection filled with 88 of these wavelengths would transport 9 terabits per second. This configuration has a typical zero margin reach of $2000 \mathrm{~km}$, a dispersion tolerance of $\pm 50000 \mathrm{ps} / \mathrm{nm}$, and a PMD tolerance of 20 ps of mean DGD. The compact spectrum can traverse twelve 


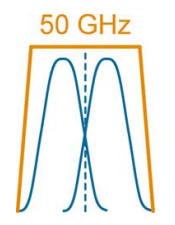

Fig. 22. Two modulated carriers spaced $20 \mathrm{GHz}$ apart within a 50-GHz ITU bandwidth.

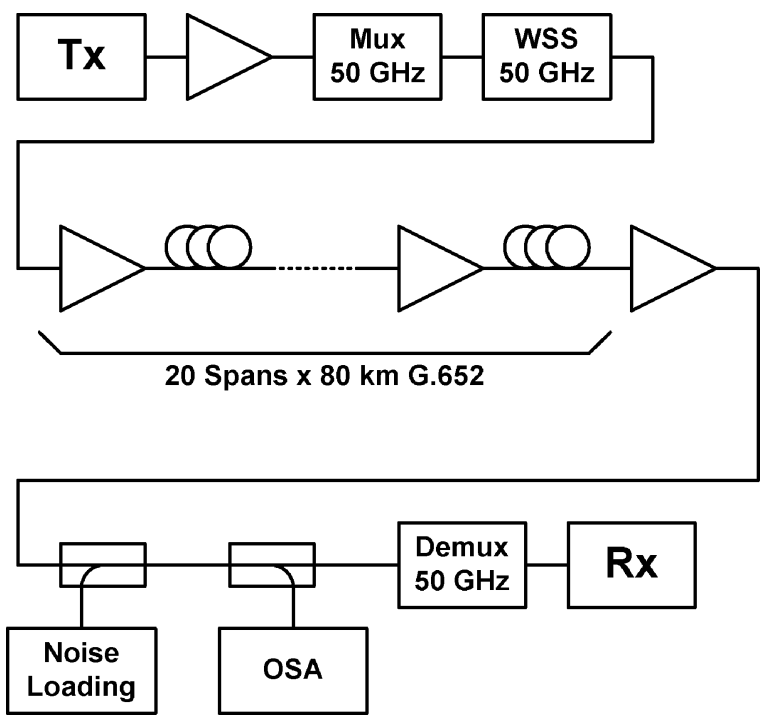

Fig. 23. Block diagram of test set up used for measurements of dual-subcarrier dual-polarization QPSK at $112 \mathrm{~Gb} / \mathrm{s}$ on 50-GHz ITU grid (single channel propagation)

WSS, with the neighboring channel locations, $50 \mathrm{GHz}$ over, set to maximum attenuation.

Single-channel propagation measurements were done on a 20-span link with G.652 fiber with different launch powers using a dual-subcarrier DP-QPSK system. The block diagram of the test set up is presented in Fig. 23 and the results are shown in Fig. 24. No in-line dispersion compensation was used. The increase in ROSNR back-to-back, as compared to the results of Fig. 17, can be explained as follows. The line rate increase from 46 to $112 \mathrm{~Gb} / \mathrm{s}$ causes a theoretical penalty of $3.9 \mathrm{~dB}$. The rest of the increase is due to hardware implementation which includes a crosstalk penalty due to sub-carrier separation of $20 \mathrm{GHz}$.

\section{SINGLE-CARRIER $100 \mathrm{~GB} / \mathrm{S}$}

With advances in the speed of CMOS circuits, the baud value for coherent transmission systems can be doubled from 14 to $28 \mathrm{Gbaud}$, allowing single carrier $112 \mathrm{~Gb} / \mathrm{s}$ transmission. This reduces cost by halving the number of components. With the large increase in the number of CMOS gates available in an ASIC, more advanced signal processing algorithms can be implemented, and so the $112 \mathrm{~Gb} / \mathrm{s}$ performance can be made to match that of the $46 \mathrm{~Gb} / \mathrm{s}$. This is important because it allows the overlay of $112 \mathrm{~Gb} / \mathrm{s}$ wavelengths on routes designed for $46 \mathrm{~Gb} / \mathrm{s}$. Fig. 25(a) shows the measured optical spectrum of a single-carrier $112 \mathrm{~Gb} / \mathrm{s}$ transmitter. Fig. 25(b) shows the recovered constellation of the same transmitter in a noise-loaded back-to-back regime at $\mathrm{BER}=3.8 \times 10^{-3}$. The experiment was performed

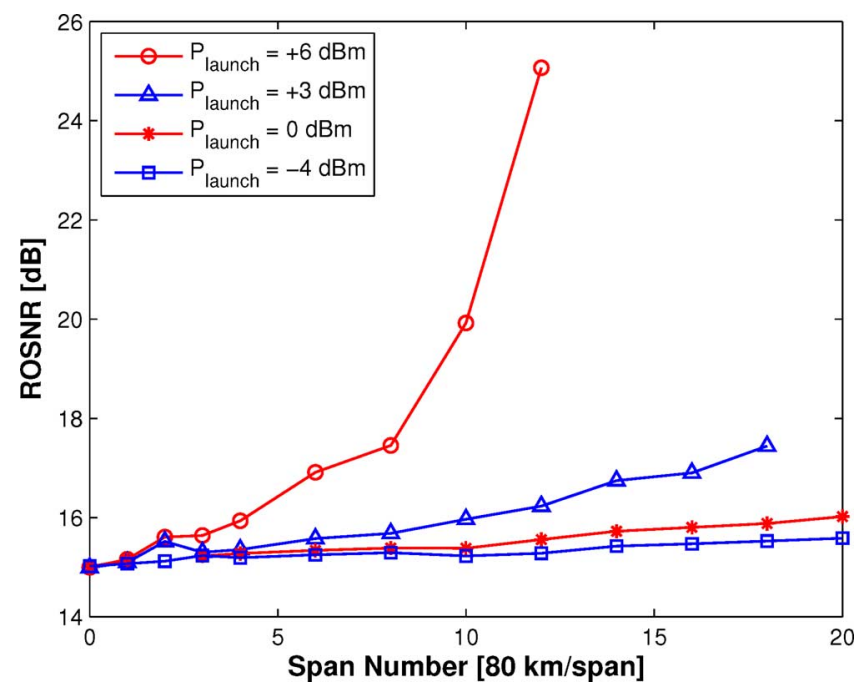

Fig. 24. ROSNR versus span count ( $80 \mathrm{~km} / \mathrm{span}, \mathrm{G} .652$ fiber) for dual-subcarrier dual-polarization QPSK system at $112 \mathrm{~Gb} / \mathrm{s}$ on $50-\mathrm{GHz}$ ITU grid (single channel propagation). Resolution bandwidth $=0.1 \mathrm{~nm}$.

back-to-back with scope capture and offline processing. Algorithms similar to the ones used at $46 \mathrm{~Gb} / \mathrm{s}$ were used.

\section{OfF-Line vs. ReAL-Time Processing}

Off-line processing is useful during the development phase of a project. It allows for development, verification, and refinement of processing algorithms. Since information is captured and stored, different algorithms can be applied and compared with the same data. Off-line processing experiments allow the refinement of models which lead to a better understanding of the propagation effects and the hardware imperfections, before the design is frozen in an ASIC. Off-line processing also permits investigation of coherent optical systems in research environments such as universities.

Unless the real-time processing hardware is especially designed to be able to receive a signal that is only a short burst, recirculating loop experiments require off-line processing. Of course, in a commercial product real-time processing is required since, in general, payload data for a high capacity optical transmission system is real-time and continuous.

Off-line experiments need to carefully treat low probability or long time duration effects in order to ensure a post-FEC bit error rate of better than $10^{-16}$. The potential error flooring or FEC punch-through effects [26] cannot be measured with feasible amounts of brute-force off-line computations. Similarly, the tails of the probability densities of polarization effects need to be explicitly explored. Transients may persist over billions of symbols which can be longer than the available capture memory. Confidence that these effects have been adequately considered only comes with long-term real-time tests.

\section{FUtURE}

Coherent transceivers operating at $46 \mathrm{~Gb} / \mathrm{s}$ are currently available and will be available at $112 \mathrm{~Gb} / \mathrm{s}$ in the very short term. Single carrier dual-polarization 16-QAM (14 Gbaud, $112 \mathrm{~Gb} / \mathrm{s}$ ) has been demonstrated [27]. We expect that coherent transmission systems operating at rates of 200,400 , and $1000 \mathrm{~Gb} / \mathrm{s}$ per 


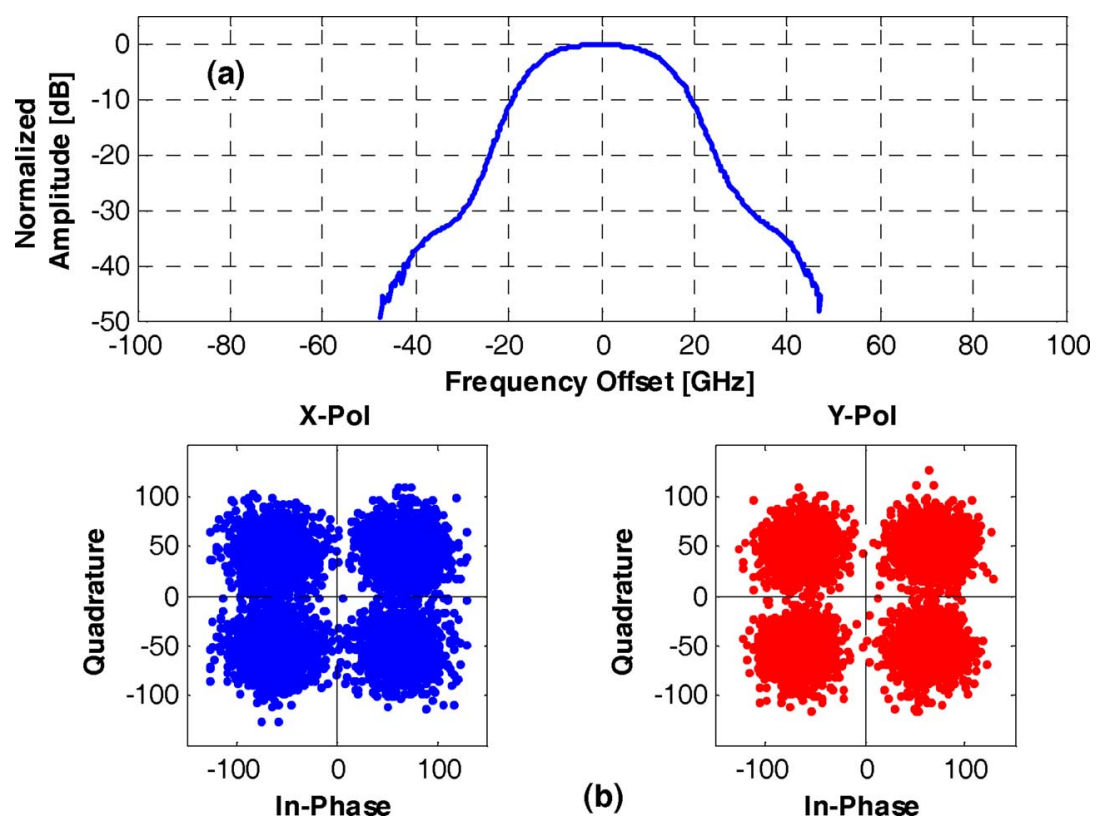

Fig. 25. Single-carrier 28 Gbaud DP-QPSK $(112 \mathrm{~Gb} / \mathrm{s})$ transmission. (a) Optical spectrum of transmitter (resolution bandwidth $=0.07 \mathrm{~nm}$ ). (b) Recovered constellation diagrams for $\mathrm{X}$ and $\mathrm{Y}$ polarizations in back-to-back regime with noise loading for $\mathrm{BER}=3.8 \times 10^{-3}$.

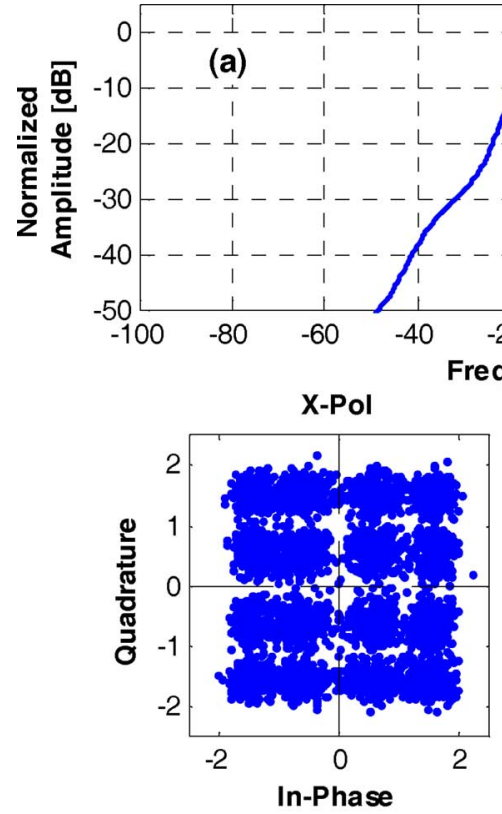

(b)

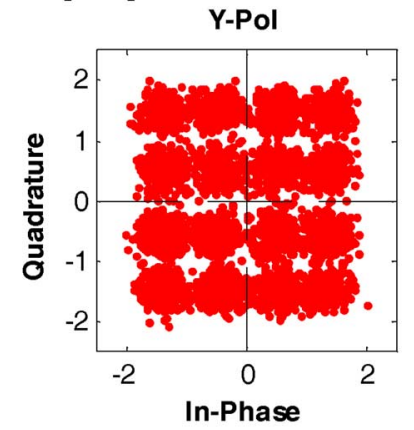

Fig. 26. Dual-polarization 16-QAM signal at 28 Gbaud ( $224 \mathrm{~Gb} / \mathrm{s}$ ). (a) Optical spectrum (resolution bandwidth $=0.07 \mathrm{~nm}$ ), (b) $16-\mathrm{QAM}$ constellations for X and Y polarizations.

wavelength will become available in the medium term. We can speculate on how this may be achieved. Since the bit rate is inherently the product of the number of bits per baud, the baud value, and the number of carriers, one or more of these dimensions will need to be dramatically increased.

Table I holds examples of more complex modulation formats that can increase the number of bits per baud. For example, we realized dual-polarization 16-QAM at 28 Gbaud with $4.48 \mathrm{bit} / \mathrm{s} / \mathrm{Hz}$ (in a $50-\mathrm{GHz}$ channel) for a total bit rate of $224 \mathrm{~Gb} / \mathrm{s}$ (Fig. 26). 128-QAM, also with dual polarization, has achieved 14 bits per baud [28]. However, there is a significant price to pay for this benefit in terms of the required implementation precision and the performance penalty. For example, more amplitude levels, such as 16-QAM and more, require more precision (i.e., more bits) from ADCs, which leads to more complex designs. Faster processing also incurs more heat dissipation.

The increased sampling frequency and massively parallel digital signal processing needed to multiply the baud value are assured by a long history of steady improvements in CMOS technology. The application of one and two sub-carriers has been described in this paper. Methods such as OFDM exploit large 
numbers of sub-carriers [29], [30]. Coherent optical sub-carriers have been demonstrated in [31]. When cost-effective, multiple carriers and coherent carrier discrimination will multiply the number of bits carried in one wavelength.

As has been described above, coherent processing can eliminate the effects of linear impairments. This leaves optical noise and nonlinearities to be dealt with. Current forward error correction and coding is $2.1 \mathrm{~dB}$ from the soft Shannon bound (at $\mathrm{R}=0.93)$. CMOS digital signal processing will be exploited to obtain some portion of this possible improvement in noise tolerance.

It was demonstrated in [32] that optical nonlinearities arising from known signals can be electrically compensated, and that the limits are due to the complexity and speed of the required hardware. CMOS improvements, and new methods, will allow future transmission systems to substantially mitigate known nonlinearities.

\section{CONClusion}

The recent commercialization of coherent optical transmission at $46 \mathrm{~Gb} / \mathrm{s}$ presently attests to the readiness of supporting technologies for use in volume manufacture. Chief amongst these is capable CMOS ASIC technology which affords the ADCs and DSP necessary for access to and manipulation of the optical electric field signal. This, to such an extent that, polarization multiplexed WDM transmission of phase modulated signals is practical over long haul distances. Further it has been shown that previously complex optical functions can be performed via DSP thereby simplifying the transceiver implementation.

We have demonstrated that for such phase modulated systems it is advantageous to perform dispersion compensation at the receiver site thereby eliminating compensators from the line. This design reduces inter-channel nonlinearities and is easily implemented in receive DSP. The marginal power required to support such compensation is estimated to be low cost.

Advances in analog and digital IC technologies have made it feasible to design and manufacture optical coherent modems. Coherent detection enables a wide choice of modulation formats, including multiple sub-carriers within a channel and the use of dual polarization. Coherent detection enables digital signal processing for equalizing various linear and nonlinear impairments. System measurement results have been shown for dual-polarization quadrature-phase-shift-keying with one and two sub-carriers, transmitting 46 and $112 \mathrm{~Gb} / \mathrm{s}$, using symbol rates of 11.5, 14, and 28 Gbaud. Laboratory measurements have been shown for dual polarization 16-QAM, transmitting $224 \mathrm{~Gb} / \mathrm{s}$. Appropriate linear filtering compensated the linear propagation effects of chromatic dispersion, polarization rotation, and polarization mode dispersion. The robust tolerance of the coherent systems to transients and propagation conditions has been demonstrated. It is anticipated that the flexibility and performance provided by optical coherent detection will enable future optical transmission systems at 200, 400, and then $1000 \mathrm{~Gb} / \mathrm{s}$ per wavelength.

\section{REFERENCES}

[1] S. Tsukamoto, D.-S. Ly-Gagnon, K. Katoh, and K. Kikuchi, "Coherent demodulation of $40-\mathrm{Gbit} / \mathrm{s}$ polarization-multiplexed QPSK signals with 16-GHz spacing after 200-km transmission," in Proc. OFC, 2005, PDP29.

[2] E. Ip, A. P. T. Lau, D. J. F. Barros, and J. M. Kahn, "Coherent detection in optical fiber systems," Opt. Express, vol. 16, no. 2, pp. 753-791, Jan. 2008.

[3] M. G. Taylor, "Coherent detection method using DSP for demodulation of signal and subsequent equalization of propagation impairments," IEEE Photon. Technol. Lett., vol. 16, pp. 674-676, Feb. 2004.

[4] C. R. S. Fludger, T. Dutel, D. van den Borne, C. Schulien, E.-D. Schmidt, T. Wuth, J. Geyer, E. De Man, G.-D. Khoe, and H. de Waardt, "Coherent equalization and POLMUX-RZ-DQPSK for robust 100-GE transmission," J. Lightw. Technol., vol. 26, no. 1, pp. 64-72, Jan. 2008.

[5] V. Curri, P. Poggiolini, A. Carena, and F. Forghieri, "Dispersion compensation and mitigation of nonlinear effects in 111-Gb/s WDM coherent PM-QPSK systems," IEEE Photon. Technol. Lett., vol. 20, pp. 1473-1475, Sep. 2008.

[6] Y. Han and G. Li, "Coherent optical communication using polarization multiple-input-multiple-output," Opt. Express, vol. 13, no. 19, pp. 7527-7534, Sep. 2005.

[7] K. Roberts, "40 Gb/s optical systems with electronic signal processing," in Proc. Lasers and Electro-Optics Society (LEOS), 2007, WFF4.

[8] See Manufacturers of Pump Lasers Bookham, JDSU, Lumics [Online]. Available: www.bookham.com, www.jdsu.com, www.lumics.com

[9] J. R. Barry and E. Lee, "Performance of coherent optical receivers," Proc. IEEE, vol. 78, pp. 1369-1394, Aug. 1990.

[10] C. Laperle, B. Villeneuve, Z. Zhang, D. McGhan, H. Sun, and M. O'Sullivan, "WDM performance and PMD tolerance of a coherent 40-Gb/s dual-polarization QPSk transceiver," J. Lightw. Technol., vol. 26, no. 1, pp. 168-175, Jan. 2008.

[11] F. M. Gardner, Phase-Locked Techniques, 2nd ed. New York: John Wiley \& Sons, 1979, pp. 8-13.

[12] E. Ip and J. Kahn, "Feedforward carrier recovery from coherent optical communications," J. Lightw. Technol., vol. 25, no. 9, pp. 2675-2692, Sep. 2007.

[13] A. J. Viterbi and A. M. Viterbi, "Nonlinear estimation of PSK-modulated carrier phase with applications to burst digital transmission," IEEE. Trans. Inf. Theory, vol. IT-29, no. 4, pp. 543-551, Jul. 1983.

[14] G. P. Agrawal, Nonlinear Fiber Optics, 2nd ed. San Diego, CA: Academic, 1995, sec. 3.2 .

[15] G. P. Agrawal, Fiber-Optic Communication Systems, 2nd ed. New York: Wiley, 1997, sec. 2.3.

[16] S. J. Savory, G. Gavioli, R. I. Killey, and P. Bayvel, "Electronic compensation of chromatic dispersion using a digital coherent receiver," Opt. Express, vol. 15, no. 5, pp. 2120-2126, Mar. 2007.

[17] T. Duthel, C. R. S. Fludger, J. Geyer, and C. Schulien, "Impact of polarization dependent loss on coherent POLMUX-NRZ-DQPSK," in Proc. OFC, 2008, OThU5.

[18] H. Sun, K.-T. Wu, and K. Roberts, "Real-time measurements of a 40 Gb/s coherent system," Opt. Express, vol. 16, no. 2, pp. 873-879, Jan. 2008.

[19] L. E. Nelson, S. L. Woodward, S. Foo, X. Zhou, M. D. Feuer, D. Hanson, D. McGhan, H. Sun, M. Moyer, M. O'Sullivan, and P. D. Magill, "Performance of a 46-Gbps dual-polarization QPSK transceiver with real-time coherent equalization over high PMD fiber," $J$. Lightw. Technol., vol. 27, no. 3, pp. 158-167, Feb. 2009.

[20] J. G. Proakis, Digital Communications, 2nd ed. New York: McGrawHill, 1989.

[21] T. Pfau, S. Hoffmann, O. Adamczyk, R. Peveling, V. Herath, M. Porrmann, and R. Noé, "Coherent optical communication: Towards realtime systems at $40 \mathrm{Gbit} / \mathrm{s}$ and beyond," Opt. Express, vol. 16, no. 2, pp. 866-872, Jan. 2008.

[22] P. M. Krummrich and K. Kotten, "Extremely fast (microsecond timescale) polarization changes in high speed long haul WDM transmission systems," in Proc. OFC, 2004, FI3.

[23] S. L. Woodward, L. E. Nelson, M. D. Feuer, X. Zhou, P. D. Magill, S. Foo, D. Hanson, H. Sun, M. Moyer, and M. O'Sullivan, "Characterization of real-time PMD and chromatic dispersion monitoring in a high-PMD 46-Gb/s transmission system," IEEE Photon. Technol. Lett., vol. 20, no. 24, pp. 2048-2050, Dec. 2008.

[24] R. Hui, Y. Wang, K. Demarest, and C. Allen, "Frequency response of cross-phase modulation in multispan WDM optical fiber systems," IEEE Photon. Technol. Lett., vol. 10, pp. 1271-1273, Sep. 1998. 
[25] G. Goeger, M. Wrage, and W. Fischler, "Cross-phase modulation in multispan WDM systems with arbitrary modulation formats," IEEE Photon. Technol, Lett., vol. 16, pp. 1858-1860, Aug. 2004.

[26] D. J. Kennedy and M. B. Nakhla, "Burst error characterization of FEC coded digital channels," Int. J. Sat. Commun., vol. 10, no. 5, pp. 243-250, 1992.

[27] P. J. Winzer and A. H. Gnauck, "112-Gb/s polarization-multiplexed 16-QAM on a 25-GHz WDM grid," in Proc. ECOC, 2008, Th.3.E.5.

[28] H. Goto, K. Kasai, M. Yoshida, and M. Nakazawa, "Polarization-multiplexed $1 \mathrm{Gsymbol} / \mathrm{s}, 128-\mathrm{QAM}$ (14 Gbit/s) coherent optical transmission over $160 \mathrm{~km}$ using a $1.4 \mathrm{GHz}$ Nyquist filter," in Proc. OFC, 2008, JThA45.

[29] N. E. Jolley, H. Kee, R. Rickard, J. Tang, and K. Cordina, "Generation and propagation of a $1550 \mathrm{~nm} 10 \mathrm{Gbit} / \mathrm{s}$ optical orthogonal frequency division multiplexed signal over $1000 \mathrm{~m}$ of multimode fibre using a directly modulated DFB," in Proc. OFC, 2005, OFP3.

[30] L. B. Du and A. J. Lowery, "Improved nonlinearity precompensation for long-haul high-data-rate transmission using coherent optical OFDM," Opt. Express, vol. 16, no. 24, pp. 19920-19925, Nov. 2008.

[31] A. D. Ellis, F. C. G. Gunning, and T. Healy, "Coherent WDM: The achievement of high information spectral density through phase control within the transmitter," in Proc. OFC, 2008, OThR4.

[32] K. Roberts, C. Li, L. Strawczynski, M. O'Sullivan, and I. Hardcastle, "Electronic precompensation of optical nonlinearity," IEEE Photon. Technol. Lett., vol. 18, pp. 403-406, Jan. 2006.

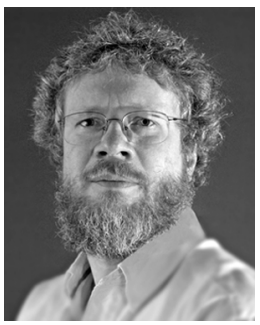

Kim Roberts (S'81-M'84) received the B.A.Sc. and M.A.Sc. degrees in electrical engineering from the University of British Columbia, Vancouver, BC, Canada.

He has innovated in the areas of optical transmission and high-capacity packet connections since 1984. His creations are at the heart of much of Nortel's optical transmission portfolio from the first OC-48 to the $40 \mathrm{~Gb} / \mathrm{s}$ DSP-assisted coherent transceiver. He has been granted 85 U.S. patents while at the Nortel labs in Edmonton, Harlow, U.K.,

and Ottawa, Canada.

Mr. Roberts is a Nortel Fellow. He received the Outstanding Engineer medal in 2008 from IEEE Canada.

Maurice O'Sullivan received the Ph.D. degree in physics (high resolution spectroscopy) from the University of Toronto, Toronto, ON, Canada,

He has worked for Nortel, Ottawa, ON, Canada, for a score of years, at first in the optical cable business, developing factory-tailored metrology for optical fiber, but, in the main, in the optical transmission business developing, modeling, and verifying physical layer designs and performance of Nortel's line and highest rate transmission product including OC-192 and MOR to eDC40G and CPL. He has been granted more than 20 patents.

Dr. O'Sullivan is a Nortel Fellow.

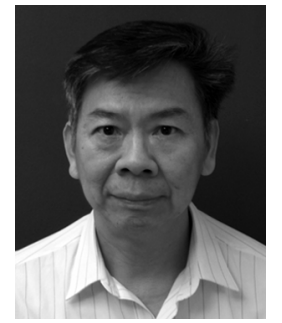

Kuang-Tsan Wu (M'82) received the B.S. and M.A.Sc. degrees from National Taiwan University (NTU), Taiwan, in 1975 and 1979, respectively, and the Ph.D. degree from the University of Ottawa, Ottawa, ON, Canada, in 1986, all in electrical engineering.

He was an instructor at the National Taiwan Institute of Technology and NTU from 1979 to 1982 . He worked on satellite modems at Microtel Pacific Research in British Columbia, Canada during 1986-87. He moved back to Ottawa in 1987 to join BNR and was the key system designer for the world's first 512-QAM digital radio. From 1994 to 1995, he had a wireless R\&D group at CCL/ITRI, Taiwan. At Nortel since then, he led the early system design of the eDC40G. He is currently applying signal processing to future optical transport systems. He has been granted nine U.S. patents and one Canadian patent.

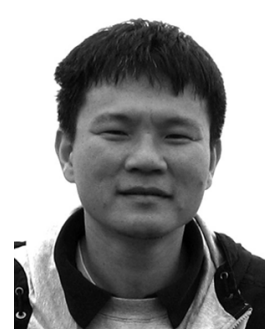

Han Sun received the B.Eng. degree in electrical engineering and the M.A.Sc. degree in photonics and semiconductor lasers from the University of Toronto, Toronto, ON, Canada, in 1997 and 1999 respectively.

He has been with Nortel, Ottawa, ON, Canada, since 2001 doing research into future optical transport systems. From 2003 onward, he has been instrumental in the development of DSP algorithms for $40 \mathrm{~Gb} / \mathrm{s}$ optical coherent modems. His research interests include application of signal processing, receiver equalization, and error correction coding to optical transmission.

Ahmed Awadalla received the B.Sc. degree in electronics and telecommunication engineering from Ain-Shams University, Cairo, Egypt, in 2003 and the M.Sc. degree in physics from the University of Waterloo, Waterloo, ON, Canada in 2007.

He is with the Nortel Networks optical R\&D team, Ottawa, ON, Canada. His current research interests are propagation impairments in optical fiber, DSP algorithms for mitigation of propagation impairments, and error correction coding.

David J. Krause (M'05) received the B.Sc. degree in electrical engineering from the University of Saskatchewan, Saskatoon, SK, Canada, in 2001 and the Ph.D. degree from Queen's University, Kingston, ON, Canada in 2007.

After a Postdoctoral Fellowship at Queen's University, he joined Nortel Networks, Ottawa, ON, Canada, in 2008.

Charles Laperle (M'90) received the B.Eng. degree from Sherbrooke University, Sherbrooke, QC, Canada in 1991, the M.Eng. degree from McMaster University, Hamilton, Ontario, Canada in 1993, and the Ph.D. degree from Laval University, Québec City, QC, Canada in 2003, all in electrical engineering.

He was with the Wireless Development Group at Nortel, Ottawa, ON, Canada from 1997 to 2000 as an RF hardware designer for TDMA wireless base stations. He has been with the Optical Research and Development Group at Nortel since 2001 as an electro-optic hardware designer. His current activities are the development of electro-optic engines and optical modems for next generation optical communication systems, and system performance evaluation. 